\title{
Study of Dementia Severity Prognosis in Mr Images Using Grey Wolf Optimization Based Dual Deep Learning Technique
}

\author{
Ahana priynaka ( $\sim$ ahanachellian@gmail.com ) \\ Madras Institute of Technology https://orcid.org/0000-0002-8487-1043 \\ Kavitha Ganesan \\ Madras Institute of Technology
}

\section{Research Article}

Keywords: Dementia, ventricle, Grey Wolf Opti- mization (GWO), Dual Deep Learning Techniques (DDLT), symmetry analysis

Posted Date: April 15th, 2021

DOI: https://doi.org/10.21203/rs.3.rs-412358/v1

License: (c) (1) This work is licensed under a Creative Commons Attribution 4.0 International License.

Read Full License 


\title{
STUDY OF DEMENTIA SEVERITY PROGNOSIS IN MR IMAGES USING GREY WOLF OPTIMIZATION BASED DUAL DEEP LEARNING TECHNIQUE
}

\author{
Ahana Priyanka Nedunchellian · Kavitha Ganesan
}

Received: date / Accepted: date

\begin{abstract}
Prognosis of in a dementia disorder is a tedious task in preclinical stage. Ventricle pathology changes in dementia appear to be overlapped for neuro degeneration in brain. Identification of these overlaps among the groups severity helps to understand the pathogenesis of this disorder. In this work impact of changes in ventricle region on severity stages of dementia is observed using dual deep learning techniques (DDLT). Alzheimer's Disease Neuroimaging Initiative (ADNI) database that contains $1169 \mathrm{MR}$ images are used in this study. Segmentation of ventricle region is carried out using multilevel threshold based Grey Wolf Optimization (GWO) technique. The feature vectors obtained from combined AlexNet and ResNet are analysed. The fused feature vectors are given to support vector machine (SVM) to observe the severity changes. Consequently, symmetry analysis of ventricle is carried out to perceive the distinctive changes in progression. The obtained results show that ventricle region is accurately delineated from other region with optimized thresholds. The segmented ventricle shows better correlation for all considered classes $(>0.9)$. It is observed that DDLT with multi class SVM provides an improved accuracy of about $79.87 \%$ compared to individual transfer learning such as AlexNet (74\%) and ResNet (76.53\%). Further, symmetry analysis shows that left side ventricle
\end{abstract}

Ahana Priyanka Nedunchellian

Department of Electronics Engineering,

Madras Institute of Technology,

Anna University, Chennai

E-mail: ahanachellian@gmail.com

Kavitha Ganesan

Department of Electronics Engineering,

Madras Institute of Technology,

Anna University, Chennai

E-mail: kavithagmit@gmail.com with DDLT features shows an improved performance than right side for onset stages. Further, clinical correlation of left ventricle seems to be statically significant $(\mathrm{p}<0.0001)$ which prominently differentiate dementia severity variations. This framework is more prominent and clinically useful to identify the distinct ventricle region variation in dementia.

Keywords Dementia · ventricle · Grey Wolf Optimization (GWO) - Dual Deep Learning Techniques $(\mathrm{DDLT}) \cdot$ symmetry analysis

\section{Introduction}

Dementia causes a rapid deterioration in cognitive ability of brain which poses serious threat to life expectancy of people. Globally 90 million people are affected by dementia and it is expected to massively increase by 2050 as given in Alzheimer's disease international report(Raza et al. 2019). The prodromal stages of this mitigating disorder are Early mild cognitive impairment (EMCI), Late mild cognitive impairment (LMCI) and Mild cognitive impairment (MCI) and Alzheimer's disease (AD). The detection of each progressive stage is difficult because of the prominent etiology and unclear overlap in severity levels (Rohini et al. 2020). The clinical diagnosis tests widely considered for dementia are Clinical Dementia Rate (CDR) and Mini mental State Examination (MMSE).These scales help to understand the rate of decline in memory where the histological progressive changes are difficult to identify.The progressive stages experience atrophy due to neuron and synapse loss. These atrophy causes the structural variation with disease progression that can be identified with the help of structural Magnetic Resonance (MR) 
images (Altaf et al. 2018). The MR effectively characterizes the structural changes that can serve as prominent biomarkers.

Numerous studies have been carried out that observe different range of atrophy in brain tissues, temporal lobes and sub-cortical regions in demented subject ( Vu et al. 2018). Change in ventricle region of brain has been observed in demented subjects (Kayalvizhi et al. 2015). The change in ventricle region is associated with various brain dysfunctions and regarded as possible biomarker for dementia. The progressive changes are sparsely investigated from early onset to severe stage. This is due to the bounded overlaps with age and structural complexity of this region (Ferrarini et al. 2006). Hence, ventricle region is considered in this study to measure the magnitude of pathology variation in demented subjects. Various studies have been attempted in literature for ventricle delineation (Liu et al. 2009). This remains challenging due to imprecise structure of brain with high in-homogenous intensity, transition regions and diffuse borders under pathology cases. Further, there is a need to understand the significance of ventricle symmetric variation under levels.

Several automated and semi-automated methods have been attempted to segment specific regions in MR images (Gonzalez-villa et al. 2016). It is found that these methods are computationally expensive, cause numerical instabilities during evolution, require adequate training and poses lower accuracy for large image size. Recently, thresholding based segmentation has been attempted to delineate the brain sub-regions because of its extensive adaptable property to define disjoint regions with closed and connected boundaries (Singh Gill et al. 2018). Further, entropy based threshold technique has been prominently used to select the threshold values. Amid them Tsallis entropy is effectively used in delineating brain regions (Nayak et al. 2019). Similarly, optimization approaches are attempted to select the effective thresholds based on nature inspired swarm intelligence algorithms. These methods provide an effective threshold with an improved search to segment the complex brain images (Chitradevi et al. 2020). In this framework, grey wolf based optimization (GWO) is considered. This method is based on hunting mechanism of wolf with leadership quality. These wolves identify the optimum position based on balanced exploration and exploitation which is suitable to handle the complex images (Khairuzzaman et al. 2017). Thus, multilevel Tsallis based GWO is employed in segmentation of complex ventricle structure.

Various report witness that brain is symmetric and identical in nature (Liu et al. 2009). The structural symmetry analysis of ventricle is considered in order to recognize the distinct changes. The existing methods for symmetry detection in brain includes deformation image warping method, intensity profile analysis, slope chain code, landmark-based symmetric plane and subspace learning techniques (Yang et al. 2009). Among them, symmetry using palindrome method is considered to produce precise left and right ventricle. This method is independent of orientation, artifacts and modality (Liang et al. 2016).

The diagnostic variation in an image is majorly identified using feature extraction methods. Exhaustive work in extraction of low level features such as shape, appearance and texture has been attempted in differentiation of normal and demented brain images. Currently high level feature extraction from an image provides an interesting observation in understanding the complex nature of diseases and its identification (Wang et al. 2020). The high level features are generally extracted using various CNN transfer learning models which poses distinct depth and number of layers to extract the features. The single learning model sometime results in vanishing gradient and reduced discriminative features.Therefore, dual deep learning methods are used in much medical application such as thoracic disease detection, chest Xray images, otoendoscopy and breast images that enhance the effectiveness of learned deep features (Chen et al. 2019; Cha et al. 2019). The complimentary dual deep learning uses AlexNet and ResNet-50. Characteristic of AlexNet such as drop out regularization and non-linearity help to extract the prominent deep features (Yuvan et al. 2016). ResNet-50 utilizes residual property to tackle the feature map losses in extraction (Talo et al. 2019; Cibuk et al. 2019). Furthermore, in medical diagnostic analysis, support vector machine (SVM) has been majorly utilized to obtain a better performance with less complexity (Ge et al. 2019).

The goal of this frame work is to study the neuro anatomical ventricle variation for normal, EMCI, MCI, LMCI and AD brain MR images based on dual deep learning features. Initially, GWO based multi-level thresholding used to delineate the ventricle regions from MR images. The pathological changes are analysed with dual deep learning features. Further, symmetry analysis of ventricle is also attempted with deep features to observe distinct difference between left and right ventricle. The extracted features from normal and severity classes are given to multi class SVM classifier. The significance test is carried out on these features and correlated with the clinical score.

The main contribution of the proposed work is described as follows. (1) This work focus on understanding the prognosis variation of ventricle for normal, EMCI, MCI, LMCI and AD using ADNI database. (2) GWO 
based multilevel thresholding is attempted for delineating ventricle region in MR images. (3) Palindrome symmetry method is used in T1 MR image to study the left and right ventricle individually in order to capture subtle changes in prognosis. (4) The effective dual deep transfer leaning model is designed and explored using AlexNet and ResNet to preserve the effectiveness of high level features of ventricle region. (5) Extensive empirical investigation on inter class variation is done on the ventricle which significantly differentiate the variation for all subjects in the database considered in this study. The paper is arranged as, section 2 summarize methods and materials followed by section 3 which elaborate result analysis finally section 3 conclude the proposed work.

\section{Methods and Materials}

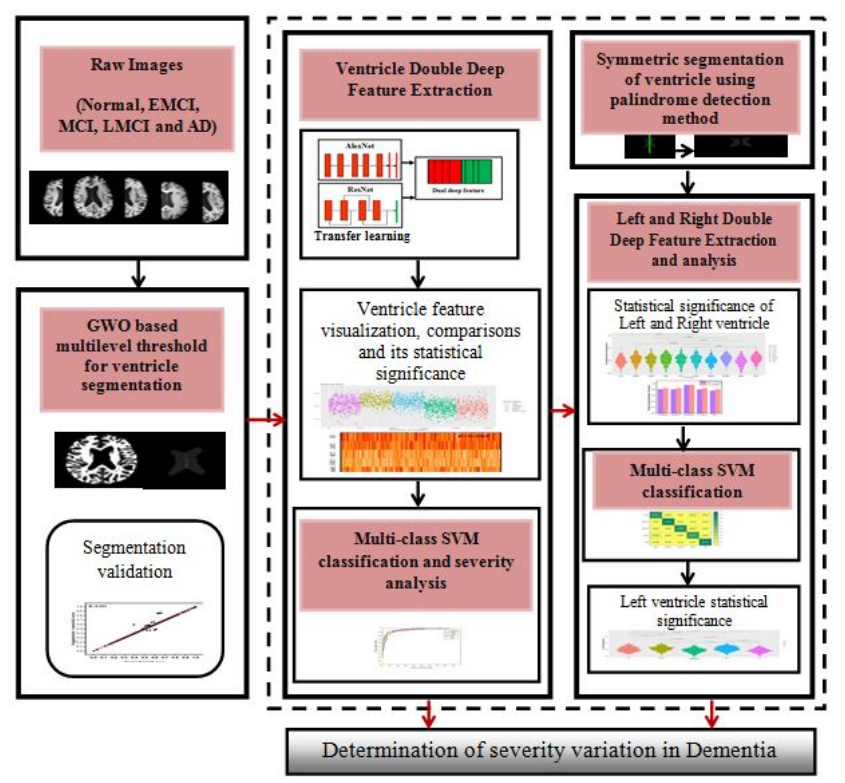

Fig. 1 Flow diagram of the proposed work

The outline of the proposed work is shown in Fig.1. The T1-weighted MR images of normal, EMCI, MCI, LMCI and AD subjects are considered for this study. Ventricle region is segmented using GWO based multilevel thresholding. Dual deep features are extracted from AlexNet and ResNet50 to observe the ventricle variations. Further, symmetry analysis of ventricle is carried out using palindrome detection method. Left and right ventricle deep feature are extracted to observe the diagnostic variations. Finally multi-class classification is performed using SVM model to assess the severity variations.The clinical score correlation and statistical significance of features are also found.

\subsection{Image database and pre-processing}

T1 weighted MR axial view images from ADNI database are considered for this analysis (Liu et al. 2020). 2500 images comprising of 229 normal, 200 EMCI, 398 MCI, $150 \mathrm{LMCI}$ and $192 \mathrm{AD}$ are chosen. The subjects are chosen based on their neuro psychological and clinical assessment. Male and female subjects are considered in this study. All MR images were resampled to 256 * $256^{*} 256$ voxels of size $1 * 1 * 1 \mathrm{~mm} 3$. Consequentially, skull stripping is performed using robust brain extraction method. This method detects the brain boundary using hybrid approach and removes the non- brain tissue to support further processing (Souza et al. 2018).

2.2 Multilevel Tsallis based threshold using grey wolf optimization

Thresholding method selects appropriate gray scale pixel values in an image to separate different intensities. Multilevel thresholding selects more threshold levels in an image to distinctively categorize the various regions. These threshold values are selected using various objective measures such as Tsallis method. This method measures the possible variations and finds the changes in the system. The existence of different states is identified by maximizing the corresponding grey level in an image. Tsallis entropy for multilevel threshold is represented as (Bhandari et al. 2015),

$T_{1}, T_{2} \ldots T_{m}=\operatorname{argmax}\left[P_{q}^{1}(t)+P_{q}^{2}(t)+. .+P_{q}^{m}(t)+(1-q) \cdot P_{q}^{1}(t) \cdot P_{q}^{2}(t) \ldots P_{q}^{m}(t)\right]$

wheret represent thresholds, $\mathrm{p}$ denote entropy, q refer to non- extensivity of the system and $\mathrm{m}$ refer to different threshold.

The optimized threshold values are obtained using GWO. This optimizer is based on the hunting behaviour of the wolf. The hunting represents the search of optimized threshold value. This optimizer consist of alpha $(\alpha)$, beta $(\beta)$, delta $(\delta)$, and $(\omega)$ omega wolves with hierarchy. The hunting is executed by three steps such as (i) tracking, chasing, and approaching, (ii) pursuing, encircling, and harassing (iii) finally attacking the prey or obtaining optimized threshold value. This hunting mechanism is continued and updated until prey is captured. The effectiveness of hunting mechanism lies in handling the exploration and exploitation problem in search space with less complexity and parameter tuning. The mathematical model of GWO to obtain the optimized threshold value is represented as (Khairuzzaman et al. 2017),

$\vec{G}=\left|\vec{X} \cdot \overrightarrow{Y_{p}}(t)-\vec{Y}(t)\right| \operatorname{and} \overrightarrow{Y(t+1)}=\overrightarrow{Y_{p}}(t)-\vec{Z}$. 
Here, $\vec{X} \vec{Z}$ are parameter vectors, t represents the iteration, dot denote element wise multiplication, $\overrightarrow{Y_{p}}$ indicate the position of the prey and $\vec{Y}$ specify the wolf position.

\subsection{Double deep transfer learning technique (DDLT)}

The fusion of AlexNet and ResNet- 50 models are attempted which help to obtain the distinct information for dementia diagnosis.

Deep learning model AlexNet: Alexnet is composed of various convolution layer, maxpool layer and fully connected layer (Ge et al.2019). This method use ReLU as an activation function to avoid gradient vanishing problem that supports non linearity in a network. This method exhibits high generalization capability due to drop out in fully connected layer.

Deep learning model ResNet-50: Resnet-50 represents the depth of the residual network (Talo et al. 2019). This model has residual block that handles the over fitting problem and loss in training phase. This residual block is utilized in order to have less parametric intervention that improve the performance of deep features. The deep feature are extracted from convolution layer which is expressed as (Yuan et al. 2016),

$C(p, q)=(M * w)(p, q)=\sum_{x} \sum_{y} M(p-x, q-y) w(x, y)$

Where $\mathrm{w}$ is the convolution kernel of size $(\mathrm{x}, \mathrm{y})$. DDLT work based on the consider asymmetric network such as AlexNet and Resnet-50. This learning helps to extract the deep feature vectors. Similarly, obtained complementary feature vectors from these asymmetric networks are fused directly to obtain the DDLT deep features.This effectively helps to summarize the pathological difference of dementia.

\subsection{Symmetry plane using palindrome detection}

Symmetric plane is search based on palindrome detection is carried out to delineate the left and right ventricle. This method utilizes linear time palindrome algorithm and RANSAC fitting to detect the symmetric axis. Initially, palindrome searching is carried out in an image with an assumption of each pixel as a string (Chen et al. 2010). The algorithm searches the palindrome in random position $\mathrm{p}$ with the radius $\mathrm{X}$. When radius of $\mathrm{X}$ is minimum there is high probability of finding the fitted palindrome. The search is done vertically and horizontally with maximum weight path to generate the symmetric axis within palindrome radii. Finally,
RANSAC helps to fit the axis in order to reduce the outliers (Liang et al. 2016).

\subsection{Support Vector machine (SVM)}

SVM is designed to classify the data based on the projection of data in high dimensional with respect to its hyperplane (Yuan et al. 2016). The estimation of hyperplane for multiclass data is obtained using kernel function. The optimum hyperplane corresponds to input feature vector which determine the classification performance. In this study, one vs all which is based on voting of desired samples which is considered to classify the onset and severity condition.

\section{Result Analysis}

The skull stripped mid-slices in axial view are considered for this analysis in normal, EMCI, MCI, LMCI and $\mathrm{AD}$ as shown in Fig 2(a). It is witnessed from the figure that this subjects poses a morphological variation in ventricle region severity levels progresses. This might be due to degeneration of surrounding tissues that causes the changes in ventricle structure.It is able to observe that the ventricle structure is concave, differs for various subjects and severity levels. Segmentation of this region is carried out using GWO based multilevel threshold. This method searches the optimized thresholds in search space with proper parameter tuning to delineate the ventricle region. Figure 2(b) and (c) represents the various segmented regions of brain tissues and segmented ventricle region using GWO with optimized threshold values.

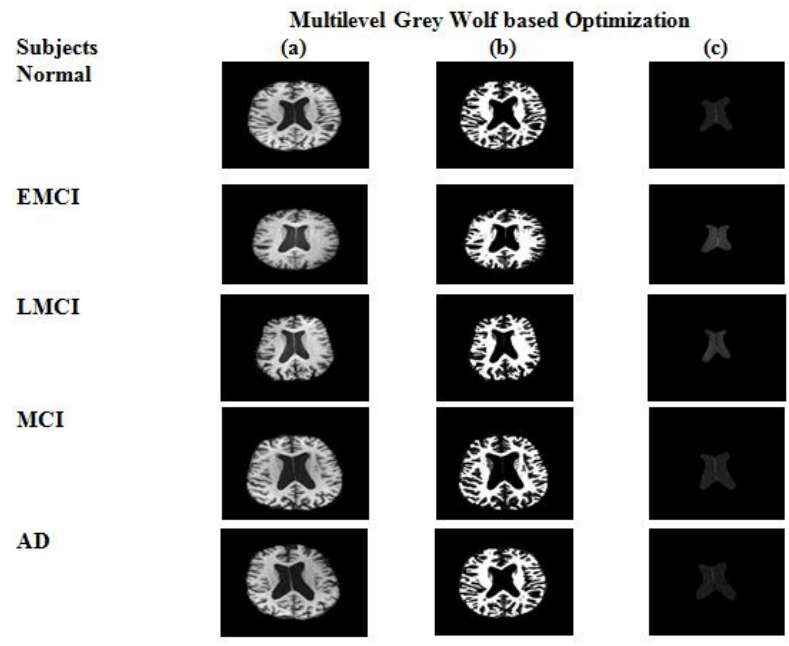

Fig. 2 Segmentation results of typical (a) Original skull stripped image (b) different threshold regions and (c) segmented ventricle region using GWO method 
Visually GWO based segmentation is able poses a finite ability to segment the complex internal brain region. The result suggests that GWO based multilevel threshold method shows improved performance in delineating the ventricle region with appropriate thresholds. However, the effectiveness of multilevel threshold based GWO is evaluated quantitatively using correlation metric for normal and severity levels. Figure 3(ae) represents the correlation of normal, EMCI, MCI, LMCI and AD ventricle areawith corresponding ground truth area. The correlation values $0.982,0.981,0.961$, 0.993 and 0.997 for normal, EMCI, MCI, LMCI and AD respectively. Thisshows that the considered method is prominently able to delineate ventricle region. The slightest variation in ground area and segmented area is due to homogenous intensity levels in some images. The obtained ventricle is considered to understand the pathology differences in onset and severity stages of dementia.

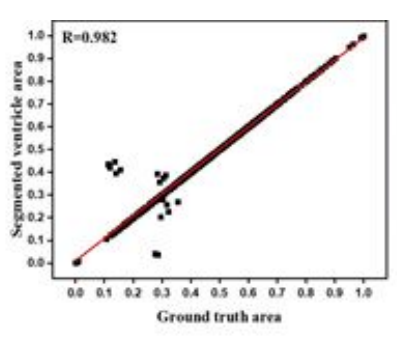

(a)

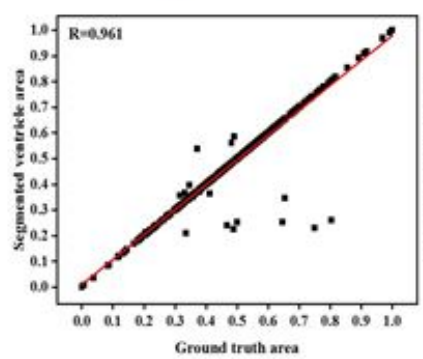

(c)

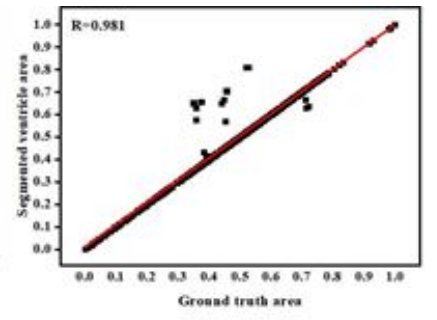

(b)

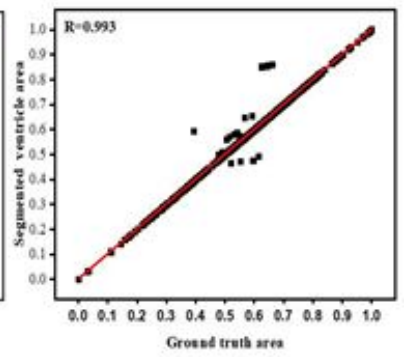

(d)

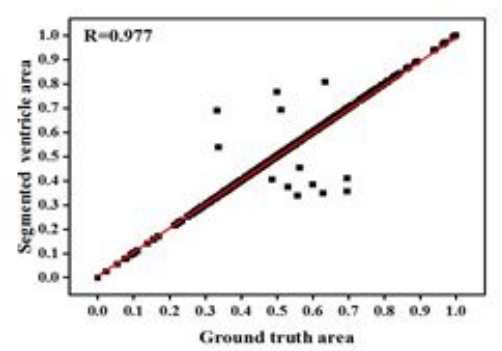

(e)

Fig. 3 Correlation of segmented ventricle of (a) Normal (b) EMCI (c) MCI (d) LMCI and (e) AD with corresponding ground truth area
The analysis of ventricle region is carried out using AlexNet, ResNet- 50 and DDLTto studyseverity variation in the abnormal images. Initially in each considered model,features are extracted. The features are obtained by tuning various parameters such as batch size $=20$, epochs $=50$ and stochastic gradient descent with momentum as a learning optimizer with 0.001 as learning rate.The considered deep learning features are extracted from the last layer of AlexNet, ResNet- 50 and DDLT that consist of 4096, 2048 and 6144 feature vectors respectively. These models extract the high level semantic deep features rather than abstract information in each class of images. This detailed deep feature helps to identify the apparent interclass variation in ventricle region for the considered class. Figure 4 shows the deep features of normal, EMCI, MCI, LMCI and AD with respect to total number of considered (2500) images. It shows that for each class of subjects, deep features extracted have different range due to variation in pattern of ventricle region.

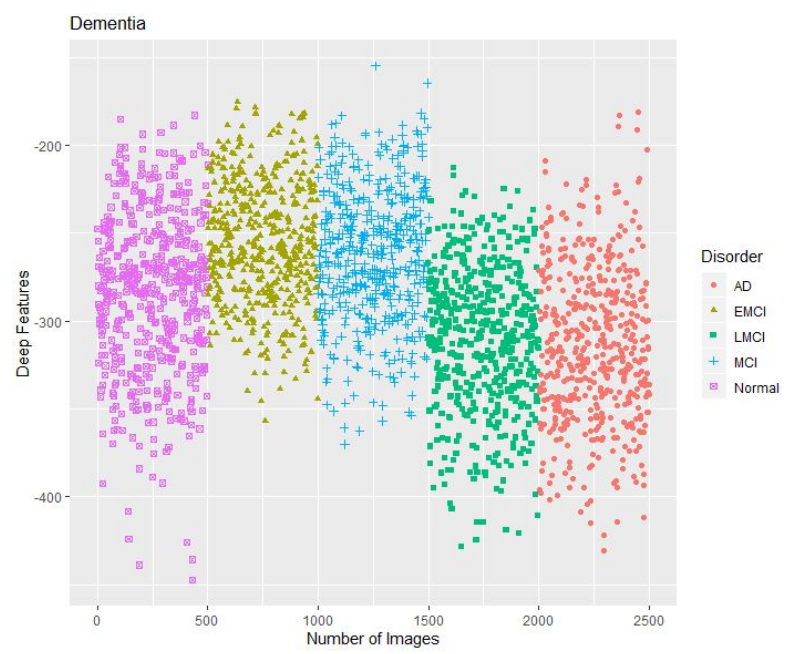

Fig. 4 Visualization of DDLT deep features for (a) Normal (b) EMCI (c) MCI (d) LMCI and (e) AD

For each feature vector extracted from AlexNet, ResNet50 and DDLT the statistical significance is analysed using ANOVA test.The ANOVA test was implemented among the considered groups with the sample size of 500 for each class and 0.05 as its significance level. Figure 5 represents the heat map p-value obtained from ANOVA test for AlexNet (0.0162), ResNet 50 (0.0748) and DDLT (0.0055).It is observed that deep features from DDLT are more statistically significant compared to AlexNet and ResNet-50. This implies DDLT feature shows a higher significance since it is able to distinguish the normal and severity classes. 


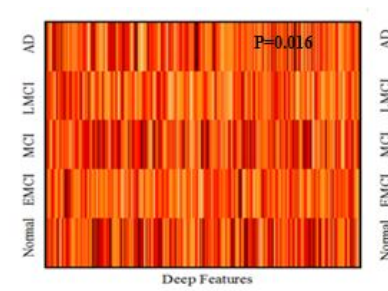

(a)

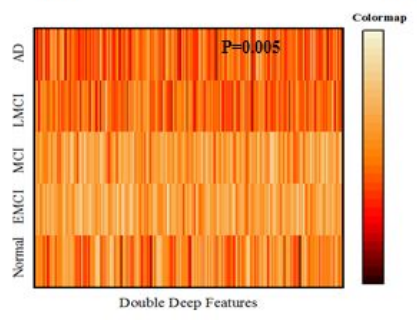

(c)

Fig. 5 Deep features heat map for considered classes and its p-values (a) AlexNet, (b) ResNetand (c) DDLT

Further, this significant features from the considered architecture is given to multiclass SVM. The classifier is used 1750 images (350 from each class) for training and 750 (150 from each class) images for testing. The classifier performance measure is depicted in Figure 6. The DDLT, AlexNet and ResNet yield an accuracy of $79.87 \%, 74 \%$ and $76.53 \%$.Sensitivity is found to be $79.7 \%, 73.9 \%, 76.51 \%$ for DDLT, Alexnet, ResNet respectively. Similarly, specificity obtained with considered methods is $93.5 \%, 94.13 \%$ and $94.97 \%$ respectively. Further, F1score of Alexnet is $74.09 \%$, ResNet- 50 is $76.08 \%$ and DDLT is $79.28 \%$. Finally, mathhhews correlation coefficient (MCC) is obtained as $0.6771,0.7162$ and 0.7592 respectively. The overall performance measure is high for DDLT than AlexNet and ResNet. This is due to the fusion of deep feature which invariably capture more distinct complementary information of pixel intensity in all considered class than AlexNet and ResNet.

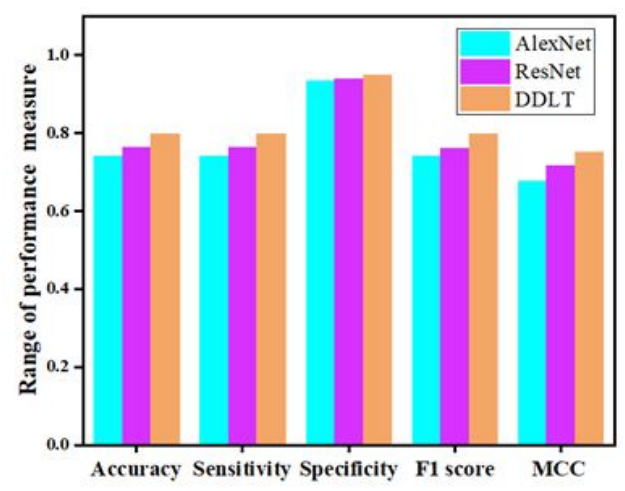

Fig. 6 Performance Measure of considered methods for ventricle region
Figure7 represents the confusion matrix of the DDLT classification outcomes. It is evident from the figure that the DDLT features are able to discriminate normal from the other categories.It can be noted that with DDLT feature set there is less overlap among EMCI, MCI, LMCI and AD. This indicates the efficacy of deep features in discriminating the degree of abnormality using ventricle region.

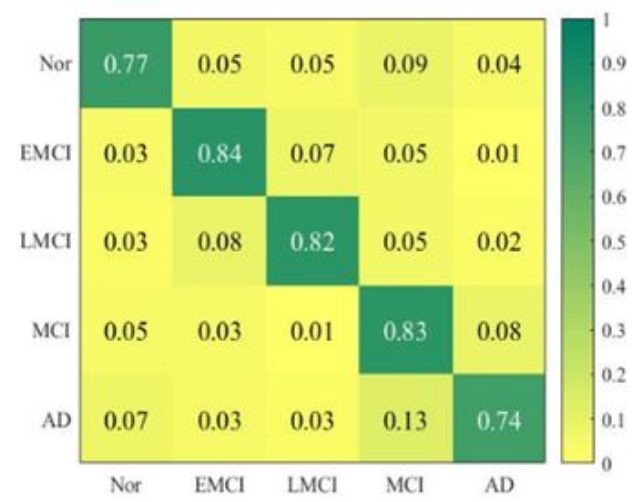

Fig. 7 DDLT method confusion matrix for whole ventricle region

Further, ROC curves for the multi-classification are obtained by macro averaging and binary prediction of true positive rate and false positive rate of considered classes. Figure 8 depicts ROC curve for each class by comparing it with all other classes.

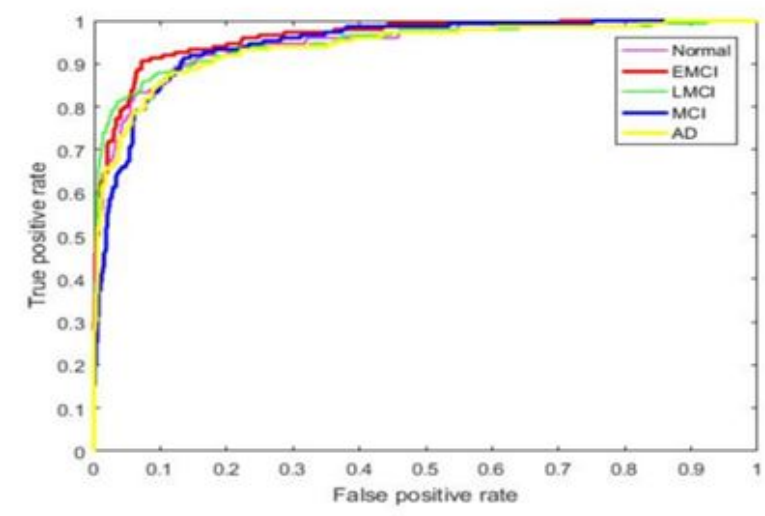

Fig. 8 ROC curve for DDLT method

The AUC for normal, EMCI, MCI, LMCI and AD are computed as $0.9483,0.9622,0.9491,0.9449$ and 0.9397 respectively. This observed result helps to predict pathological variations reliably. 
Table 1 represents the significance of DDLT deep features in terms of performance measures. Ventricular abnormalities as dementia progress help in differentiating different class. The substantial structural variation is observed between EMCI and LMCI in ventricle region that is revealed using DDLT features. MCI changes in ventricle region might be due toprogressive alteration in tissues which is reflected in deep features. Consequently, the change in $\mathrm{AD}$ is modest due to its rate of atrophy in ventricle. However, DDLTfeatures are quantitatively show an absolute difference in ventricle region for normal, EMCI, MCI, LMCI and AD.

Table 1 DDLT method performance measure of normal and severity class for ventricle region

\begin{tabular}{|c|c|c|c|c|c|}
\hline Measures & Normal & EMCI & MCI & LMCI & AD \\
\hline Accuracy & 0.917 & 0.928 & 0.933 & 0.9 & 0.918 \\
Sensitivity & 0.766 & 0.84 & 0.82 & 0.826 & 0.74 \\
Specificity & 0.955 & 0.95 & 0.961 & 0.918 & 0.963 \\
F1 score & 0.787 & 0.824 & 0.831 & 0.767 & 0.784 \\
\hline
\end{tabular}

Further, left and right ventricle region are obtained with the symmetry line palindrome detector method. Figure 9 (a-d) shows that symmetry line is precisely detected and the resultant left and right ventricles for normal, EMCI, MCI, LMCI and AD is obtained. Visual observations show that left and right ventricles exhibit changes in its structure. This could be due to the fact that ventricle structure alters when there is atrophy in cellular level in a brain. Further, DDLT features are extracted from left and right ventricle for normal and pathological images to observe the subtle morphological alterations.

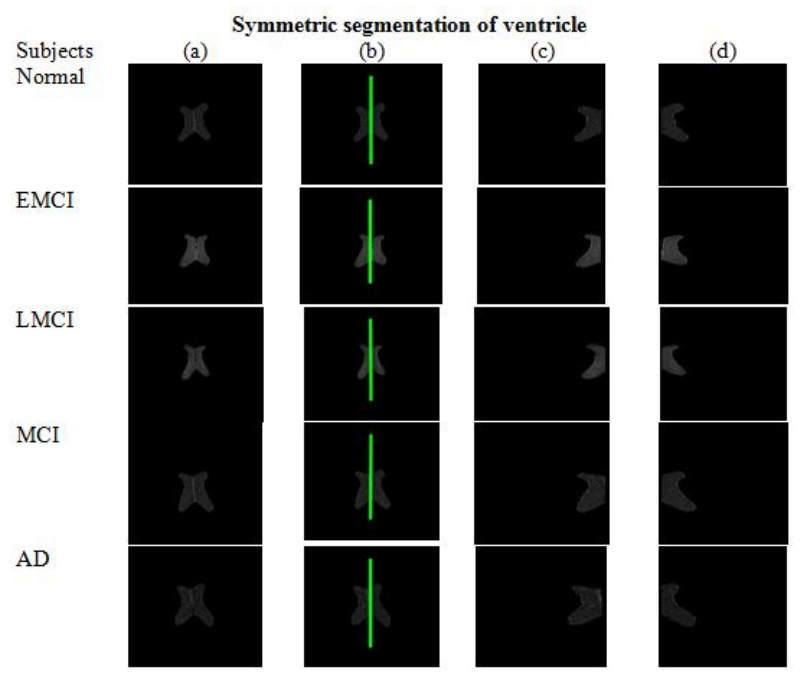

Fig. 9 Symmetric segmentation of ventricle (a) Ventricle region (b) Symmetric axis (c) Left ventricle and(d) Right ventricle
After extraction of features, potential difference between left and right ventricle are evaluated as shown in Figure 10. It is able to identify that there exist a strong difference between left and right ventricle in case of normal and pathological images $(\mathrm{p}<0.0001)$. This indicates left and right ventricle show a relative variation at different stages of severity.

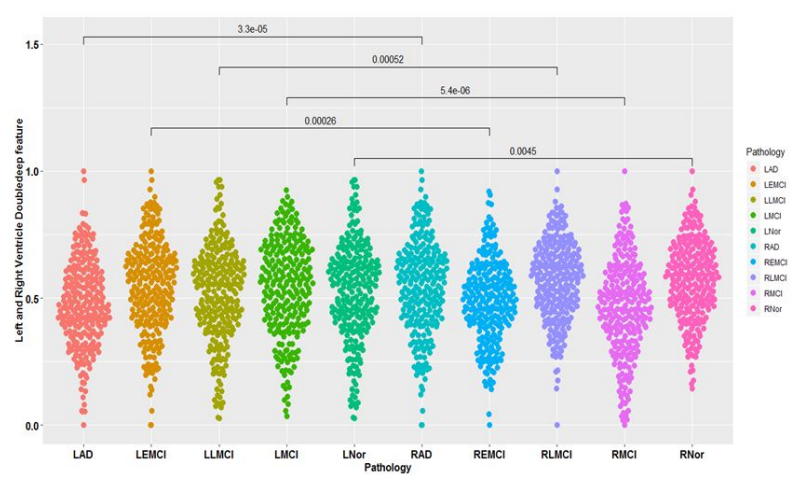

Fig. 10 Significant analysis of left and right ventricle

Furthermore, the variation in left and right ventricle is examined using multiclass SVM. The performance measures of the classifier shows that left ventricle $(84.8 \%)$ is more discriminative than right ventricle $(81.2 \%)$ as shown in Figure 11 . This could be due to the fact that degeneration related to atrophy starts from left ventricle then subsequently extends to right ventricle, is better captured using DDLT features.

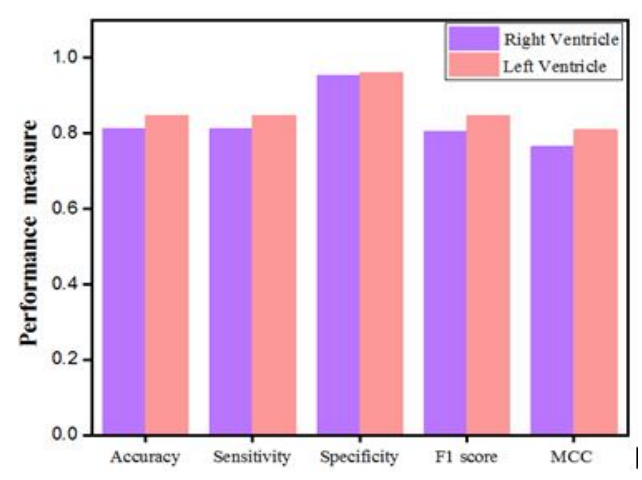

Fig. 11 Double deep feature performance measure for left and right ventricle

The confusion matrix for DDLT based SVM for left ventricleis represented in Figure 12. It shows that EMCI, LMCI and AD are prominently classified. MCI is overlapped with normal, EMCI and LMCI in classification, this might be due to similar histological changes through 
the whole extent of the left ventricle.

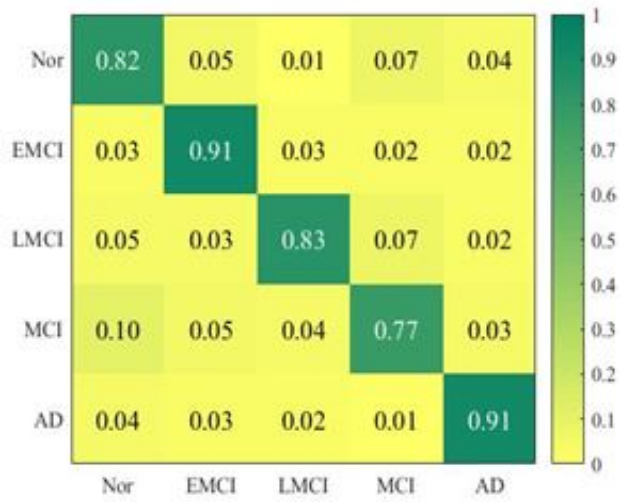

Fig. 12 Confusion matrix for "Left" ventricle using DDLT method

The area under curvature (AUC) of each ROC is computed for each class from the Figure 13 for left ventricle. The AUC values are 0.929, 0.974, 0.967, 0.955, 0.977 for normal, EMCI, MCI, LMCI and AD respectively. The DDLT based SVM model for left ventricle outperformed other multiclass models for disease classification.

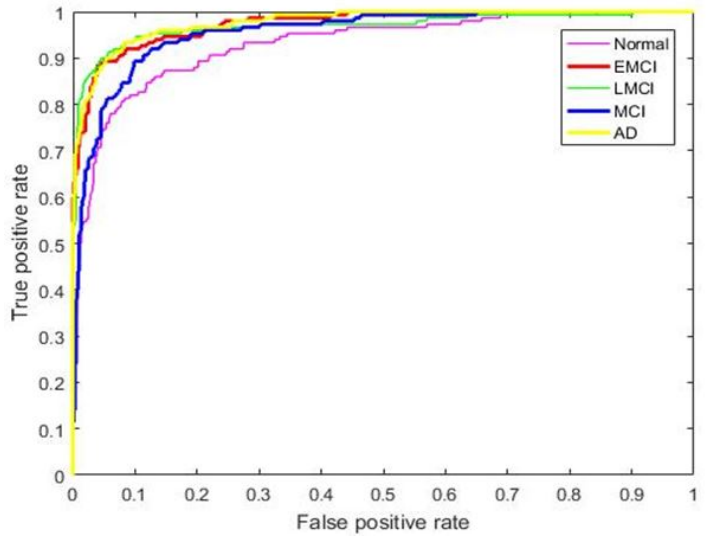

Fig. 13 ROC curve for "Left" ventricle using DDLT method

The individual class performance measure for left ventricle is represented in Table 2 . It is observed that each class shows a desirable performance to identify the severity. It demonstrates that the classification results have been improved for left ventricle than whole ventricle. This finding might indicate that change in relative size of the left ventricle is more prominent than absolute size of the ventricle.
Table 2 Performance measure of normal and severity class for "Left" ventricle driven DDLT method

\begin{tabular}{|c|c|c|c|c|c|}
\hline Measures & Normal & EMCI & MCI & LMCI & AD \\
\hline Accuracy & 0.921 & 0.948 & 0.921 & 0.946 & 0.958 \\
Sensitivity & 0.82 & 0.906 & 0.773 & 0.833 & 0.906 \\
Specificity & 0.946 & 0.958 & 0.958 & 0.975 & 0.971 \\
F1 score & 0.806 & 0.874 & 0.797 & 0.862 & 0.897 \\
\hline
\end{tabular}

The interclass variation for left ventricle is examined using ANOVA test. Figure 14 represents a left ventricle interclass variation between normal and severity classes. This helps to understand the atrophy on its onset. The left ventricle strongly correlate for each stage normal, EMCI, MCI, LMCI and AD with statistical significance $(p<0.0001)$.Thus, left ventricle can be considered as a reference to discriminate the intermediate and severity stages.

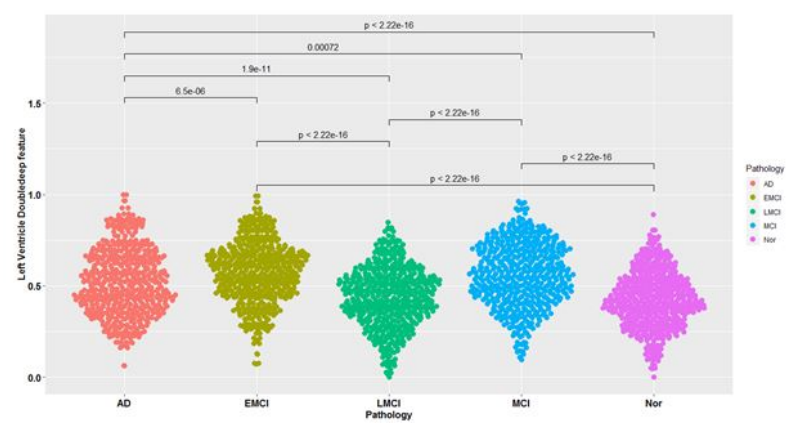

Fig. 14 Statistical analysis of "Left" ventricle in normal and various severity levels

The extracted DDLT features from left ventricle are further correlated with MMSE to observe the clinical discrimination among normal, EMCI, MCI LMCI and AD which is shown in Figure 15(a-e) respectively. DDLT features for Normal, MCI and LMCI are statistically significant with $(\mathrm{p}<0.10)$. This indicates that these features could be constructive in distinguishing between onset and severity condition in left ventricle region. Thus, this region could be considered as a biomarker in discrimination of normal and severity classes.

\section{Conclusion}

The focus of the study is to understand the diagnostic variation in normal, EMCI, MCI, LMCI and AD ventricle using DDLT features effectively. In this study, brain MR images are obtained using ADNI database for the considered classes. Ventricle region is segmented using GWO based multi-level threshold method. The proposed approach effectively delineates the complex sub 
cortical ventricle structure and poses a strong correlation with gold standards. Further, ventricle region deep features are extracted from AlexNet, ResNet and DDLT for all classes. Among them DDLT based deep features with SVM prominently shows the normal, EMCI, MCI, LMCI and AD difference in terms of statistical and performance measures for with an accuracy of $79.87 \%$. The ventricle region is further validated with symmetry analysis using palindrome detection method. It shows that left ventricle shows a distinct difference between normal and severe classes than right ventricle using DDLT features. In addition to that, clinical correlation of left ventricle DDLT feature depicts a reliable correlation with considered classes. The constructive performance of the proposed framework, it can be used to demarcate the ventricle pathology variation in EMCI, MCI, LMCI stages effectively. Hence, it could be helpful for the clinicians to understand the prognosis and enhance the preclinical assessment.

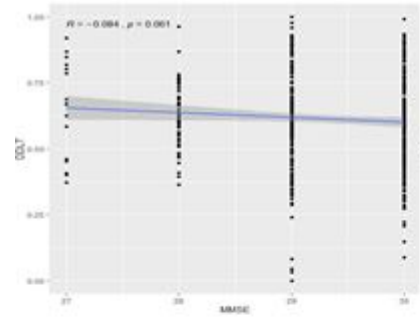

(a)

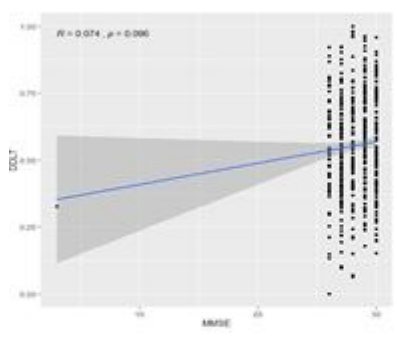

(c)

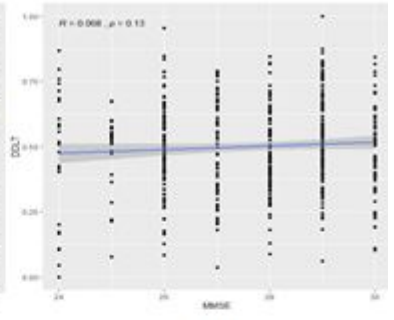

(b)

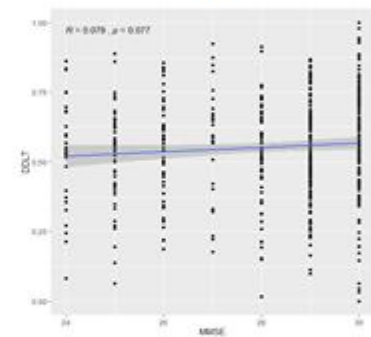

(d)

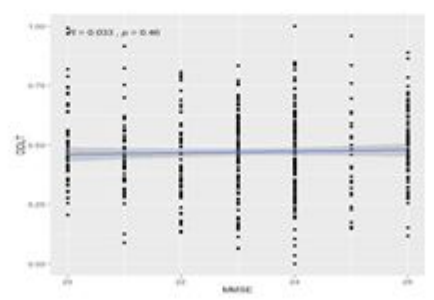

(e)

Fig. 15 Clinical correlation of DDLT for (a) normal (b) EMCI (c) MCI (d) LMCIand (e) AD

\section{Compliance with ethical standards}

Conflict of interest: The authors declare that they have no conflict of interest.
Funding: Not Applicable

Declaration: Not Applicable

Authors' contributions: Ahana priyanka Nedunchellian: Conceptualization, Data Collection, Formal analysis, Validation and Writing original draft. Kavitha Ganesan: Supervision, visualization, Writing - review and editing.

\section{References}

1. Altaf T, Anwar SM, Gul N, Majeed MN, Majid M (2018) Multi-class Alzheimer's disease classification using image and clinical features. Biomed Signal Process Control 43: 64-74. https://doi.org/10.1016/j.bspc.2018.02.019

2. Bhandari AK, Kumar A, Singh GK (2015) Tsallis entropy based multilevel thresholding for colored satellite image segmentation using evolutionary algorithms. Expert Syst Appl 42(22):8707-8730. https://doi.org/10.1016/j.eswa.2015.07.025

3. Cha D, Pae C, Seong SB, Choi JY, Park HJ (2019) Automated diagnosis of ear disease using ensemble deep learning with a big otoendoscopy image database. EBioMedicine 45: 606-614. https://doi.org/10.1016/j.ebiom.2019.06.050

4. Chen J, Moon YS, Wong MF, Su G (2010) Palmprint authentication using a symbolic representation of images. Image Vision Comput 28(3):343-351. https://doi.org/10.1016/j.imavis.2009.06.004

5. Chen B, Li J, Guo X, Lu G (2019) DualCheXNet: dual asymmetric feature learning for thoracic disease classification in chest X-rays. Biomed Signal Proces 53: 101554. https://doi.org/10.1016/j.bspc.2019.04.031

6. Chitradevi D, Prabha S (2020) Analysis of brain sub regions using optimization techniques and deep learning method in Alzheimer disease. Appl Soft Comput 86: 105857. https://doi.org/10.1016/j.asoc.2019.105857

7. Cibuk M, Budak U, Guo Y, Cevdet Ince M, Sengur A (2019) Efficient Deep Features Selections and Classification for Flower Species Recognition. Measurement 137: 713. https://doi.org/10.1016/j.measurement.2019.01.041

8. Ferrarini L, Palm WM, Olofsen H, van Buchem MA, Reiber JHC, Admiraal-Behloul, F (2006) Shape differences of the brain ventricles in Alzheimer's disease. NeuroImage 32(3): 1060-1069. https://doi.org/10.1016/j.neuroimage.2006.05.048

9. Ge C, Qu Q, Yu-Hua Gu I, Jakola AS (2019) Multi-Stream Multi-Scale Deep Convolutional Networks for Alzheimer's Disease Detection using MR Images. Neurocomputing 30: 30-39. https://doi.org/10.1016/j.neucom.2019.04.023

10. González-Villà S, Oliver A, Valverde S, Wang L, Zwiggelaar R, Lladó X (2016) A review on brain structures segmentation in magnetic resonance imaging. Artif Intell Med 73: 45-69. https://doi.org/10.1016/j.artmed.2016.09.001

11. Kayalvizhi M, Kavitha G, Sujatha CM, Ramakrishnan S (2015) Minkowski functionals based brain to ventricle index for analysis of $\mathrm{AD}$ progression in MR images. Measurement 74: 103-112. https://doi.org/10.1016/j.measurement.2015.06.021

12. Khairuzzaman AKM, Chaudhury S (2017) Multilevel thresholding using grey wolf optimizer for image segmentation. Expert Syst Appl 86: 64-76. https://doi.org/10.1016/j.eswa.2017.04.029

13. Liang S, Chen J, Li Z, Bai G (2016) Linear time symmetric axis search based on palindrome detection. In: Pro- 
ceedings of the IEEE International Conference on Image Processing (ICIP). IEEE, Arizona, pp. 1799-1803. https://doi.org/10.1109/icip.2016.7532668

14. Liu J, Huang S, Nowinski WL (2009) Automatic Segmentation of the Human Brain Ventricles from MR Images by Knowledge-Based Region Growing and Trimming. Neuroinformatics 7(2): 131-146. https://doi.org/ 10.1007/s12021009-9046-1

15. Liu SX (2009) Symmetry and asymmetry analysis and its implications to computer-aided diagnosis: A review of the literature. J Biomed Inform 42(6):1056-1064. https://doi.org/10.1016/j.jbi.2009.07.003

16. Liu M, Li F, Yan H, Wang K, Ma Y, Shen L, $\mathrm{Xu} \mathrm{M}$, for the Alzheimer's Disease Neuroimaging Initiative (2020) A multi-model deep convolutional neural network for automatic hippocampus segmentation and classification in Alzheimer's disease. NeuroImage. https:// doi.org/10.1016/j.neuroimage.2019.116459

17. Nayak DR, Dash R, Majhi B, Acharya UR (2019) Application of fast curvelet Tsallis entropy and kernel random vector functional link network for automated detection of multiclass brain abnormalities. Comput Med Imag Grap 77: 101656. https://doi.org/10.1016/j.compmedimag.2019.101656

18. Raza M, Awais M, Ellahi W, Aslam N, Nguyen HX, Le-Minh H (2019) Diagnosis and Monitoring of Alzheimer's Patients Using Classical and Deep Learning Techniques. Expert Syst Appl 136: 353-364. https://doi.org/10.1016/j.eswa.2019.06.038

19. Rohini P, Sundar S, Ramakrishnan S (2020) Differentiation of early mild cognitive impairment in brainstem MR images using multifractal detrended moving average singularity spectral features. Biomed Signal Process Control 57:101780. https://doi.org/10.1016/j.bspc.2019.101780

20. Singh Gill H, Singh Khehra B, Singh A, Kaur L (2018) Teaching-learning-based optimization algorithm to minimize cross entropy for Selecting multilevel threshold values. Egypt Inform J 20:11-25. https://doi.org 10.1016/j.eij.2018.03.006

21. Souza R, Lucena O, Garrafa J, Gobbi D, Saluzzi M, Appenzeller S, Rittner L , Frayne R, Lotufo, R (2018) An open, multi-vendor, multi-field-strength brain MR dataset and analysis of publicly available skull stripping methods agreement. NeuroImage 170: 482-494. https:// doi.org/10.1016/j.neuroimage.2017.08.021

22. Talo M, Yildirim O, Baloglu UB, Aydin G, Acharya UR (2019) Convolutional neural networks for multi-class brain disease detection using MRI images. Comput Med Imag Grap 78: 101673. https://doi.org/10.1016/j.compmedimag.2019.101673

23. Wang $\mathrm{P}$, Song Q, Li Y, Lv S, Wang J, Li L, Zhang H (2020) Cross-task extreme learning machine for breast cancer image classification with deep convolutional features. Biomed Signal Proces 57:101789. https://doi.org/10.1016/j.bspc.2019.101789

24. Vu TD, Ho NH, Yang HJ, Kim J, Song HC (2018) Nonwhite matter tissue extraction and deep convolutional neural network for Alzheimer's disease detection. Soft Comput. 22: 6825-6833https://doi.org/10.1007/s00500-018-3421-5

25. Yang W, Zhang S, Chen Y, Li W, Chen Y (2009) Shape symmetry analysis of breast tumors on ultrasound images. Comput Biol Med 39(3): 231-238. https://doi.org/10.1016/j.compbiomed.2008.12.007

26. Yuan ZW, Zhang J (2016) Feature extraction and image retrieval based on AlexNet. Eighth International Conference on Digital Image Processing (ICDIP 2016). China, https://doi.org/10.1117/12.2243849 

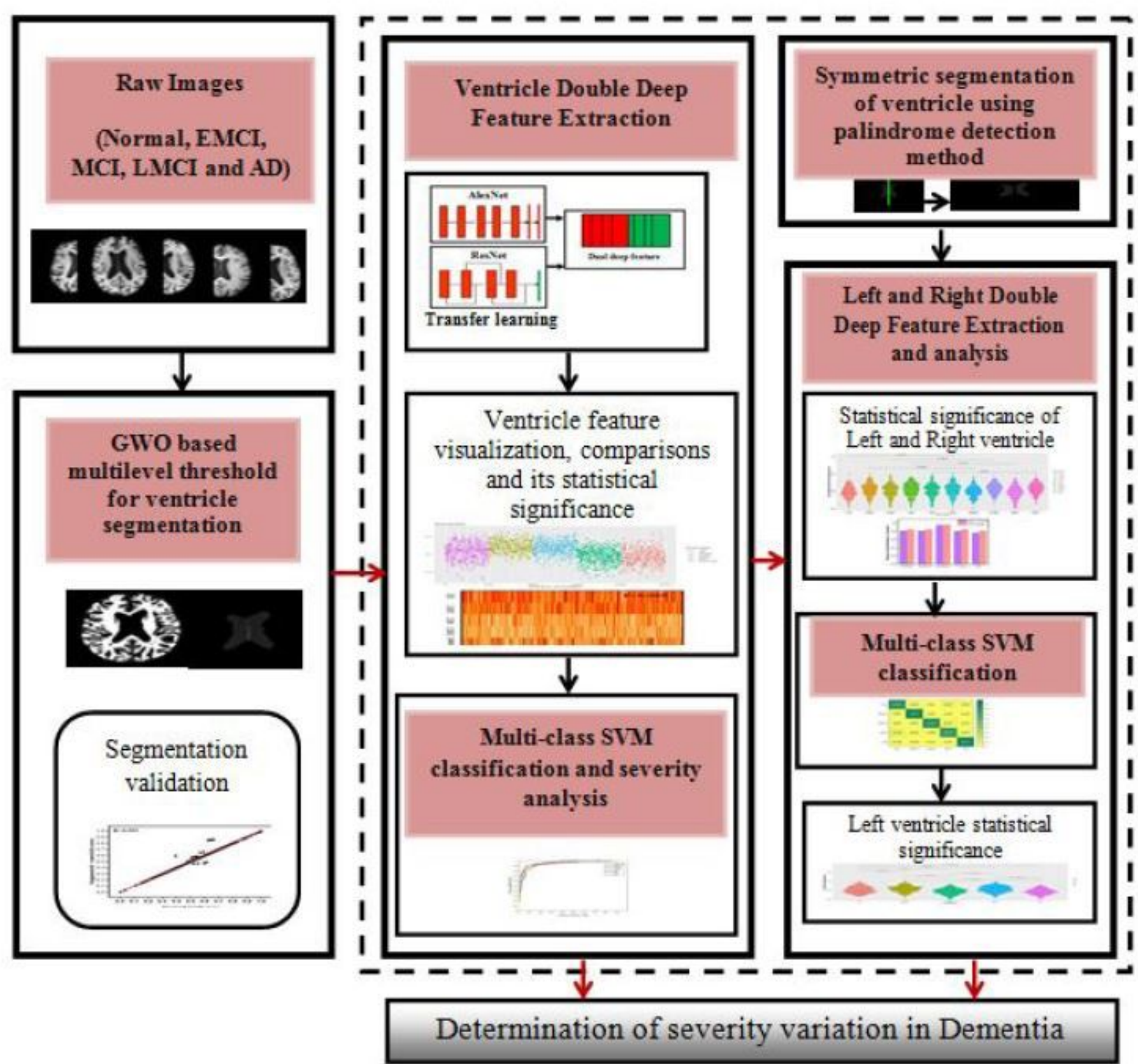

Figure 1

Flow diagram of the proposed work 
Subjects

Normal

(a)

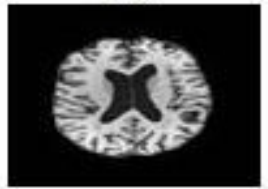

EMCI

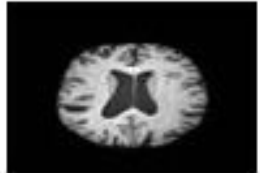

LMCI

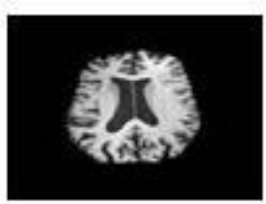

MCI

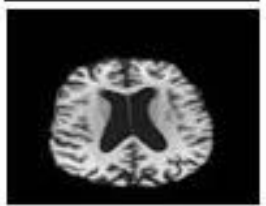

AD (b)
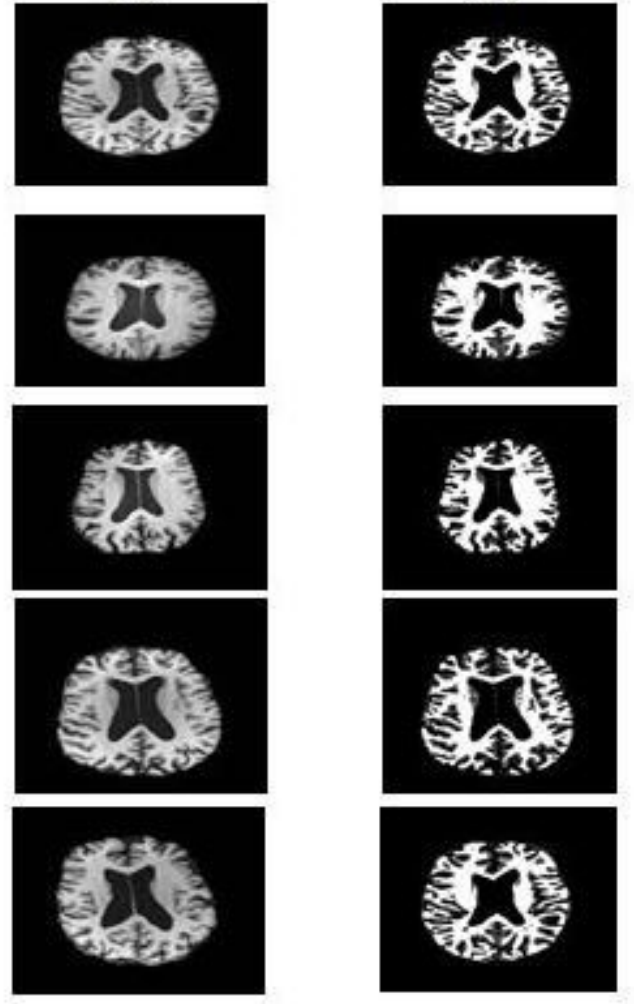

(c)
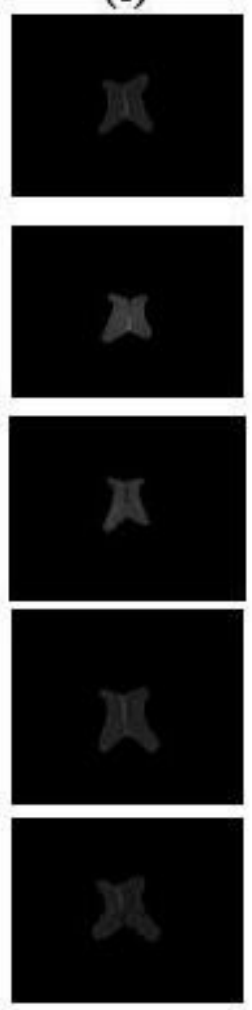

Figure 2

Segmentation results of typical (a) Original skull stripped image (b) different threshold regions and (c) segmented ventricle region using GWO method 


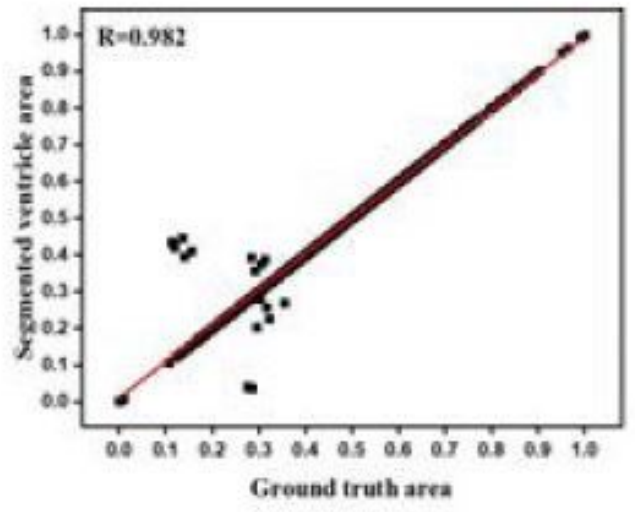

(a)

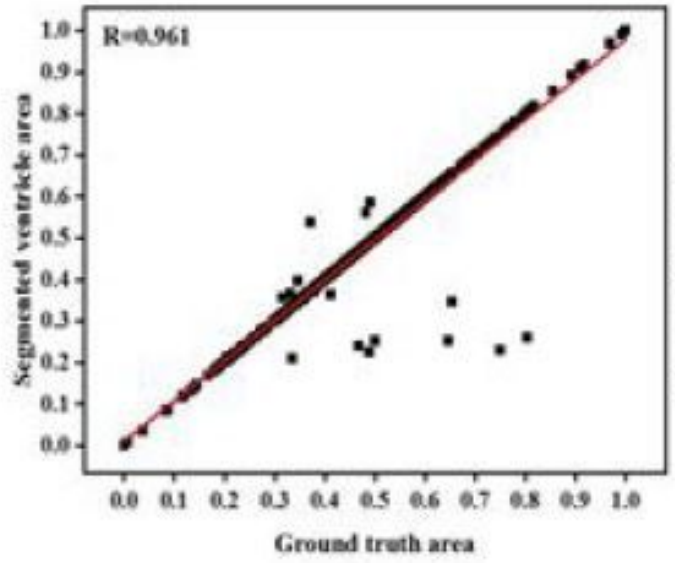

(c)

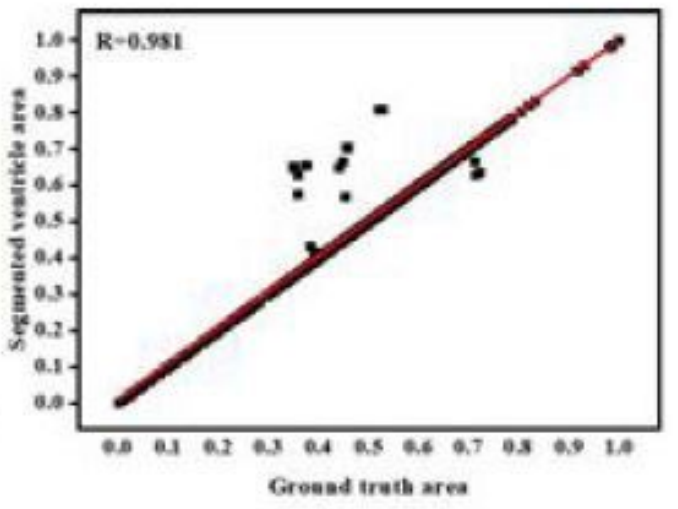

(b)

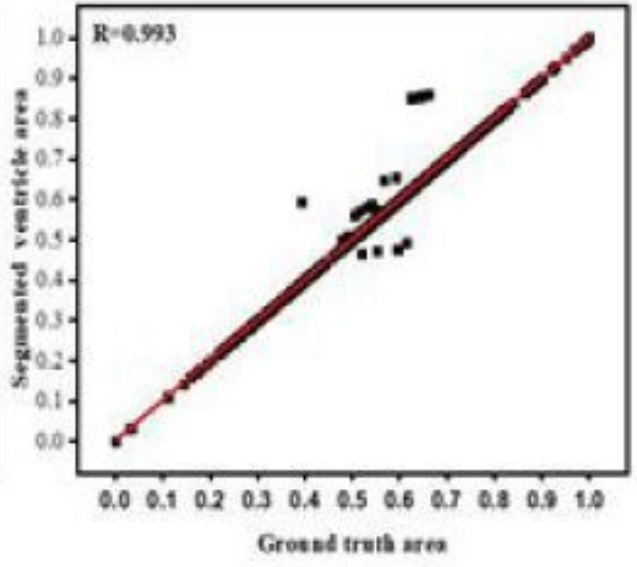

(d)

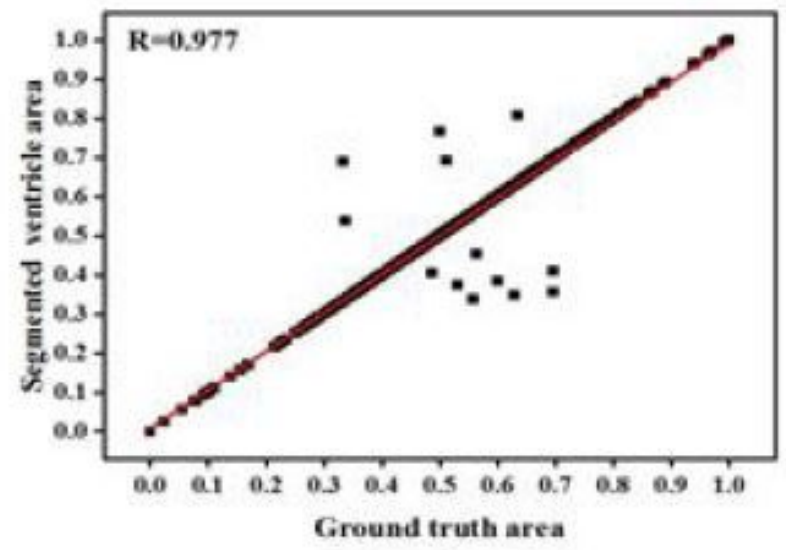

(e)

\section{Figure 3}

Correlation of segmented ventricle of (a) Normal (b) EMCl (c) MCl (d) $L M C l$ and (e) AD with corresponding ground truth area 


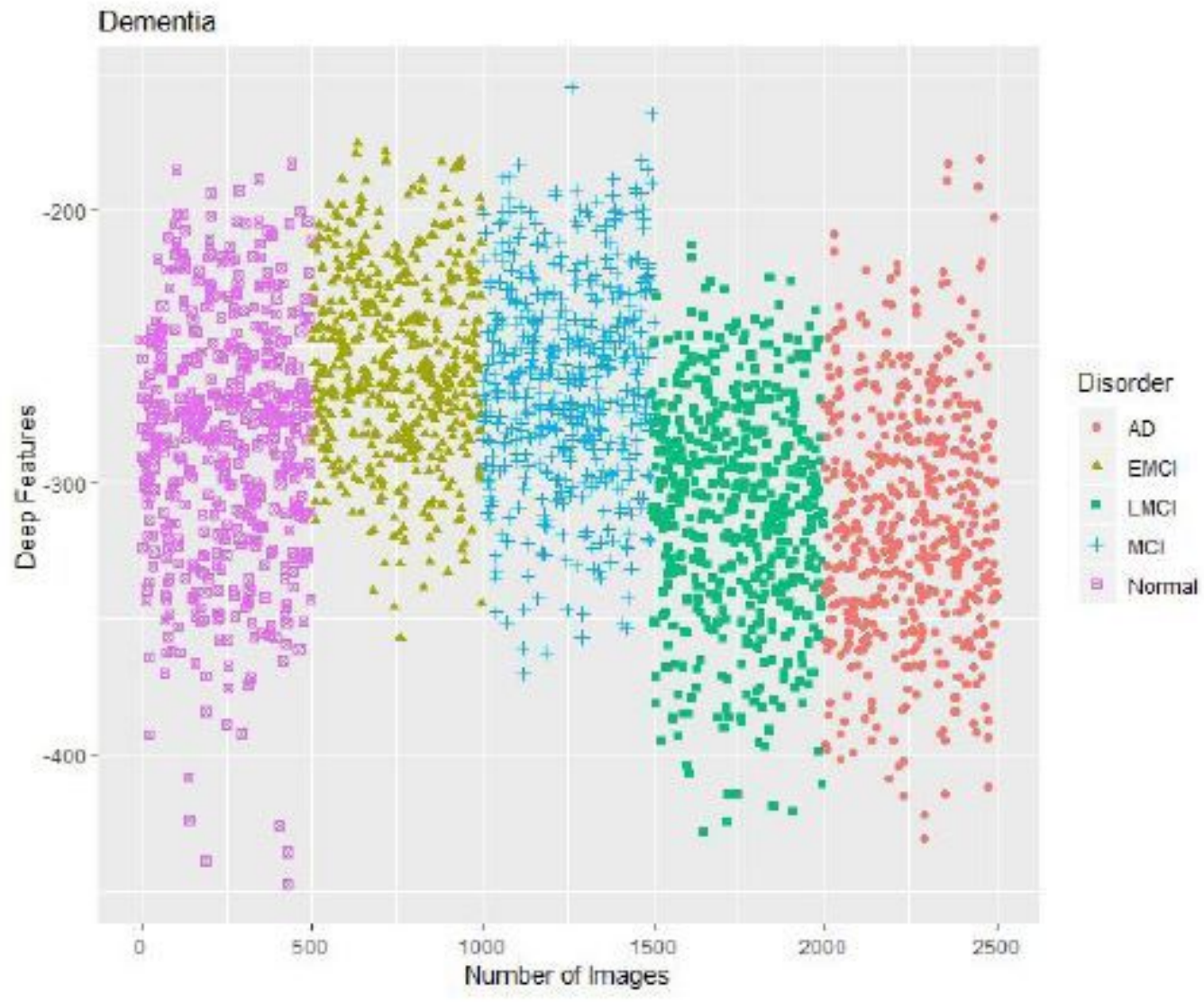

Figure 4

Visualization of DDLT deep features for (a) Normal (b) EMCl (c) MCl (d) $L M C l$ and (e) $A D$ 


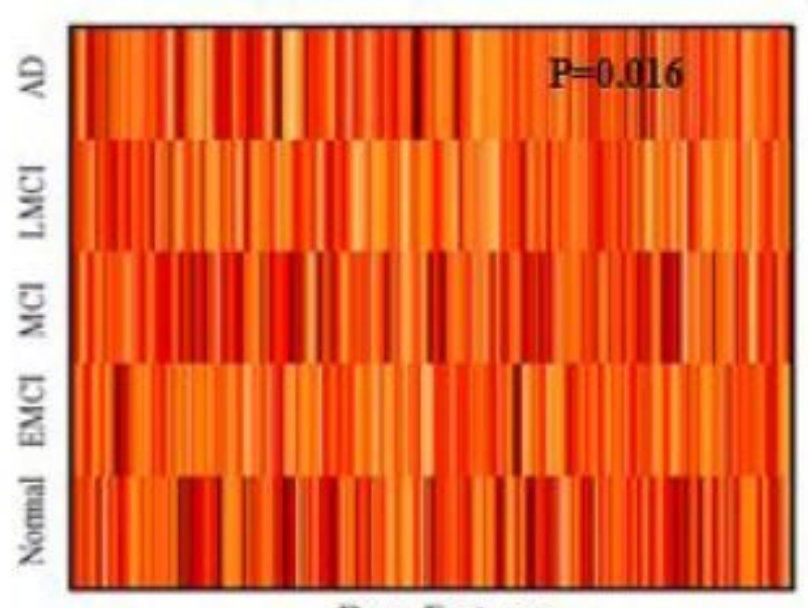

Deep Features

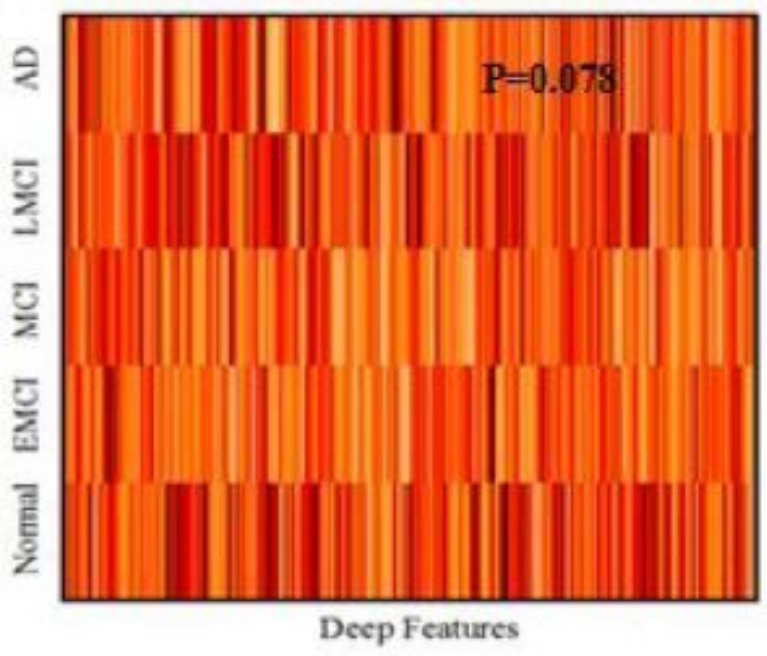

(b)

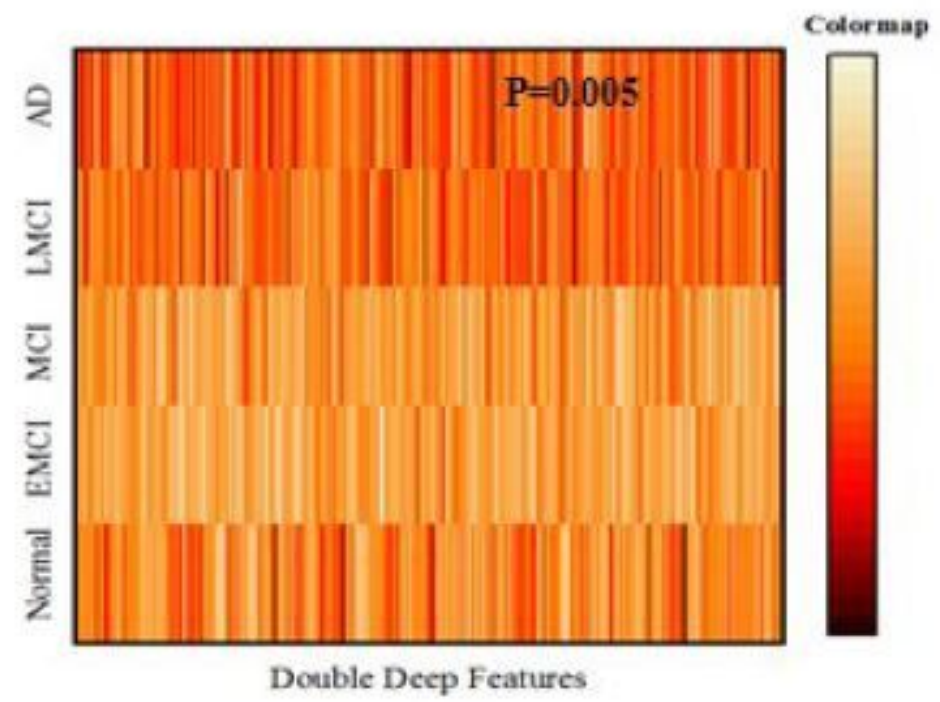

(c)

Figure 5

Deep features heat map for considered classes and its p-values (a) AlexNet, (b) ResNetand (c) DDLT 


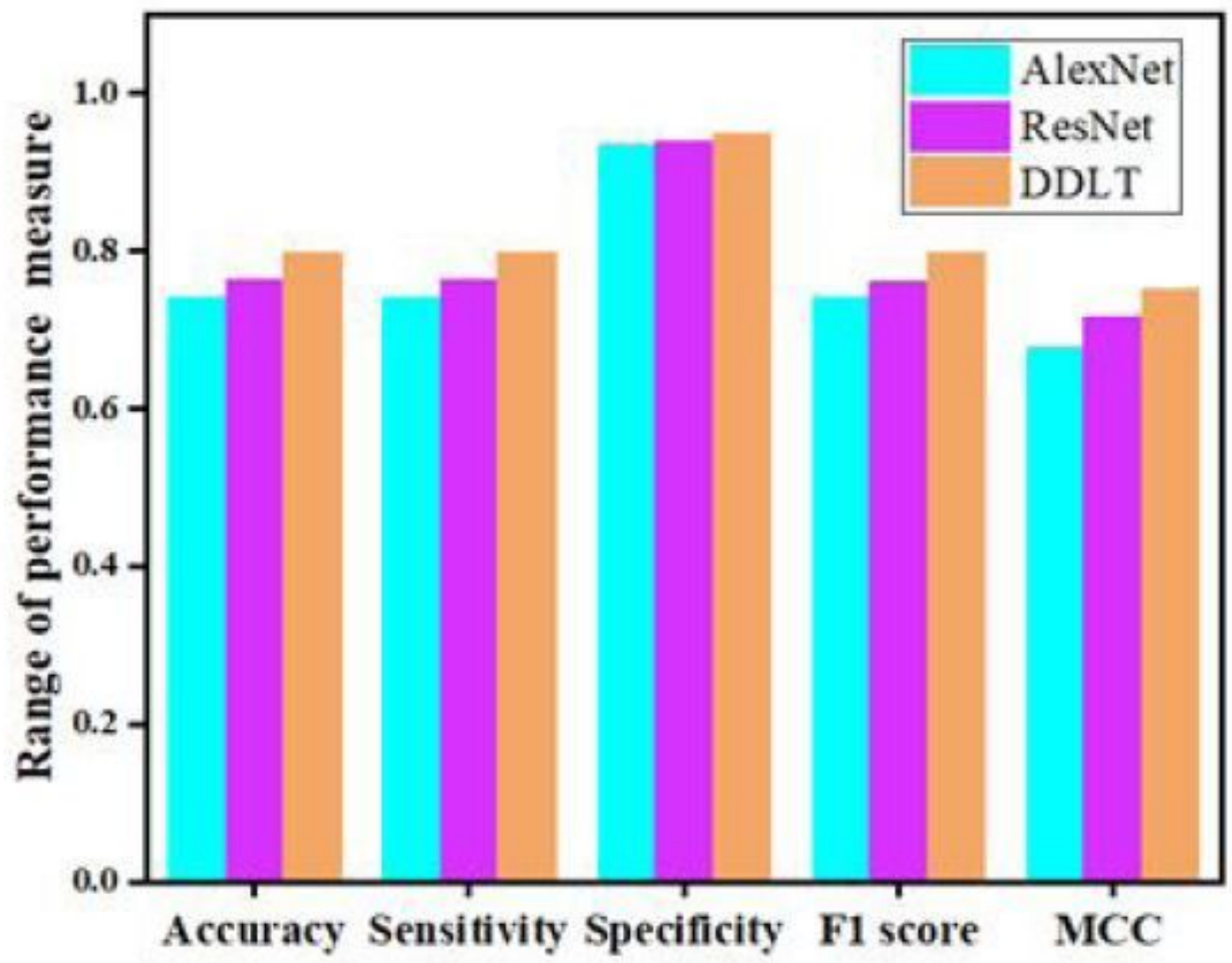

Figure 6

Performance Measure of considered methods for ventricle region 


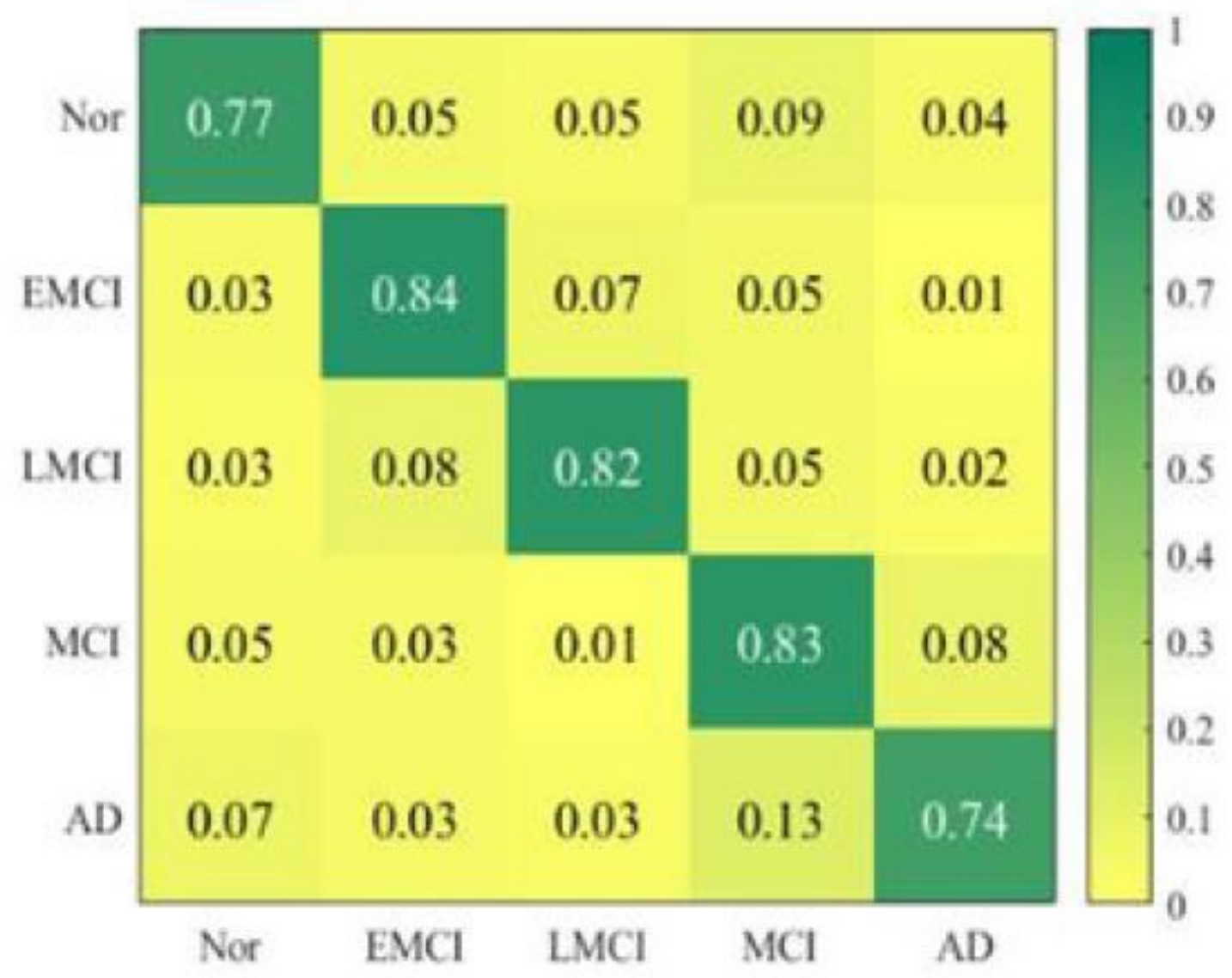

Figure 7

DDLT method confusion matrix for whole ventricle region 


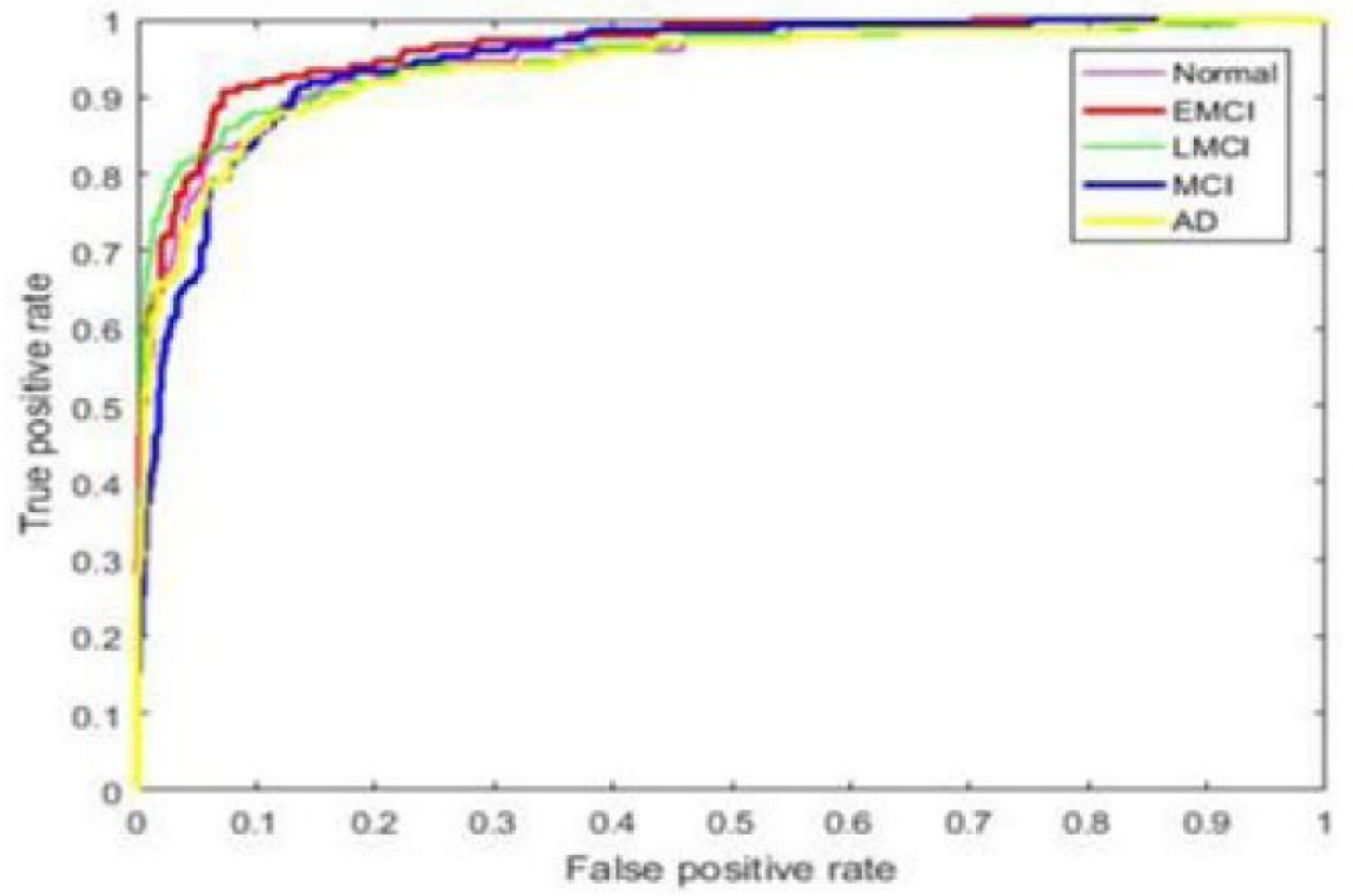

Figure 8

ROC curve for DDLT method 


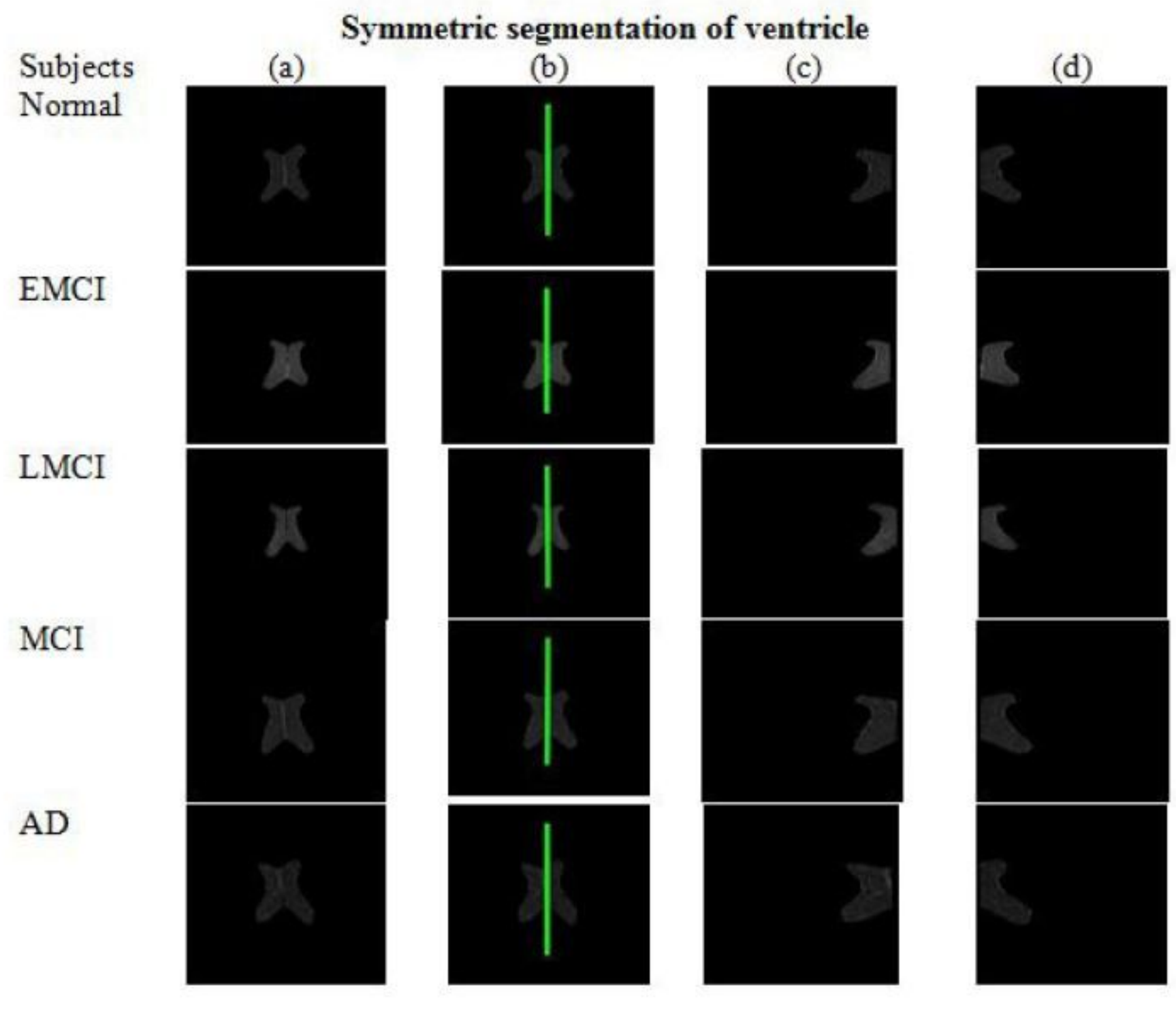

Figure 9

Symmetric segmentation of ventricle (a) Ventricle region (b) Symmetric axis (c) Left ventricle and(d) Right ventricle 


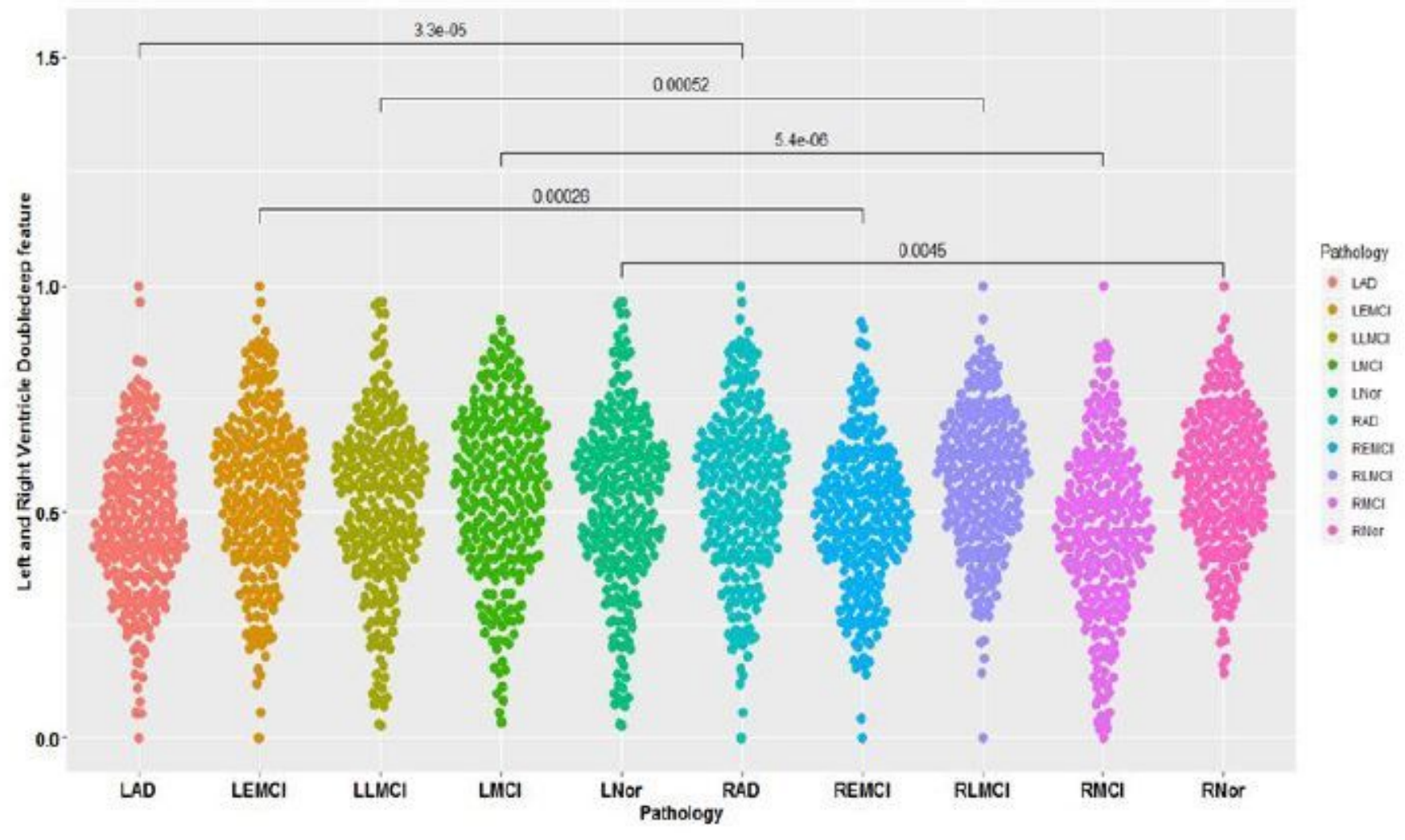

Figure 10

Significant analysis of left and right ventricle 


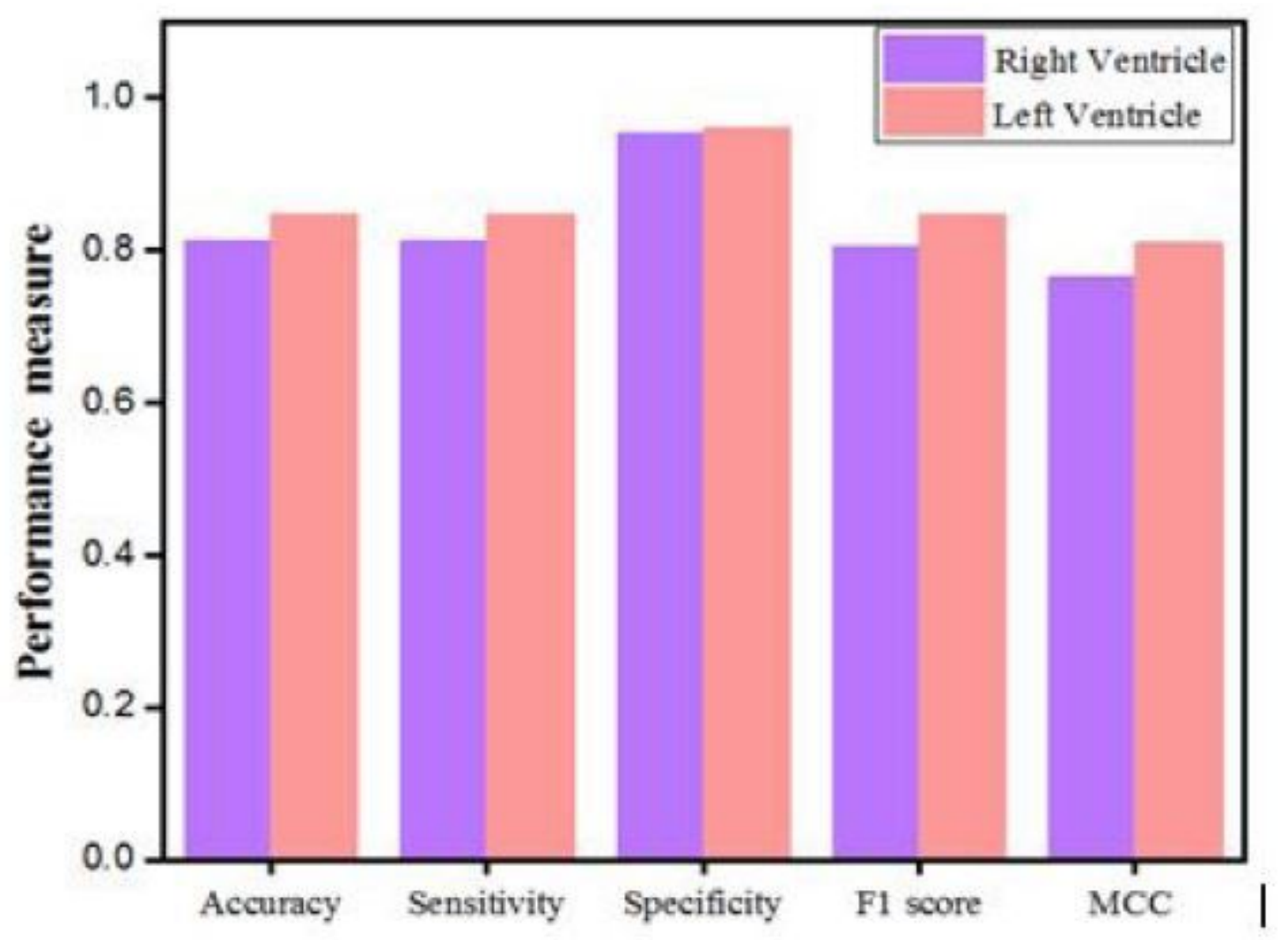

Figure 11

Double deep feature performance measure for left and right ventricle 


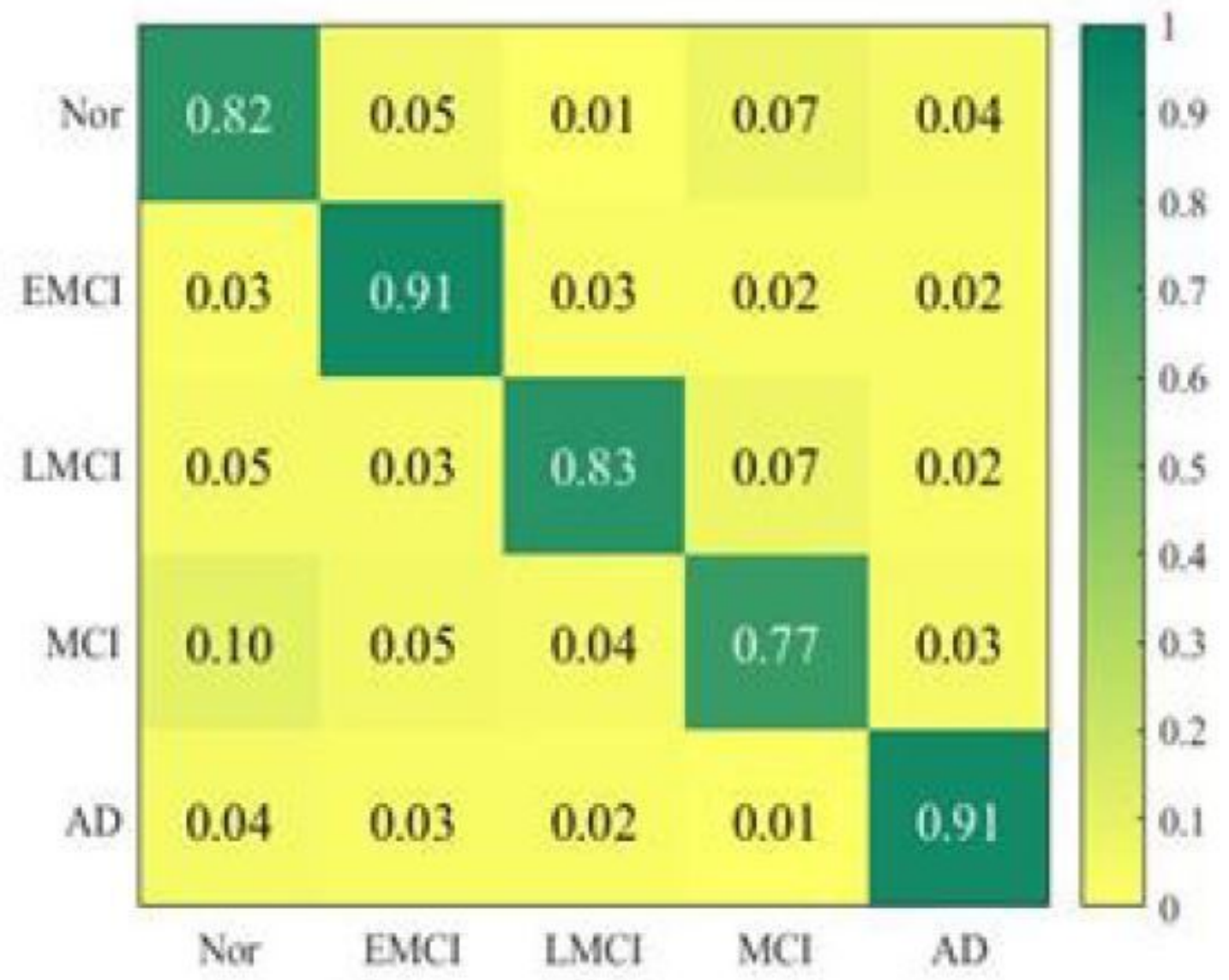

Figure 12

Confusion matrix for "Left" ventricle using DDLT method 


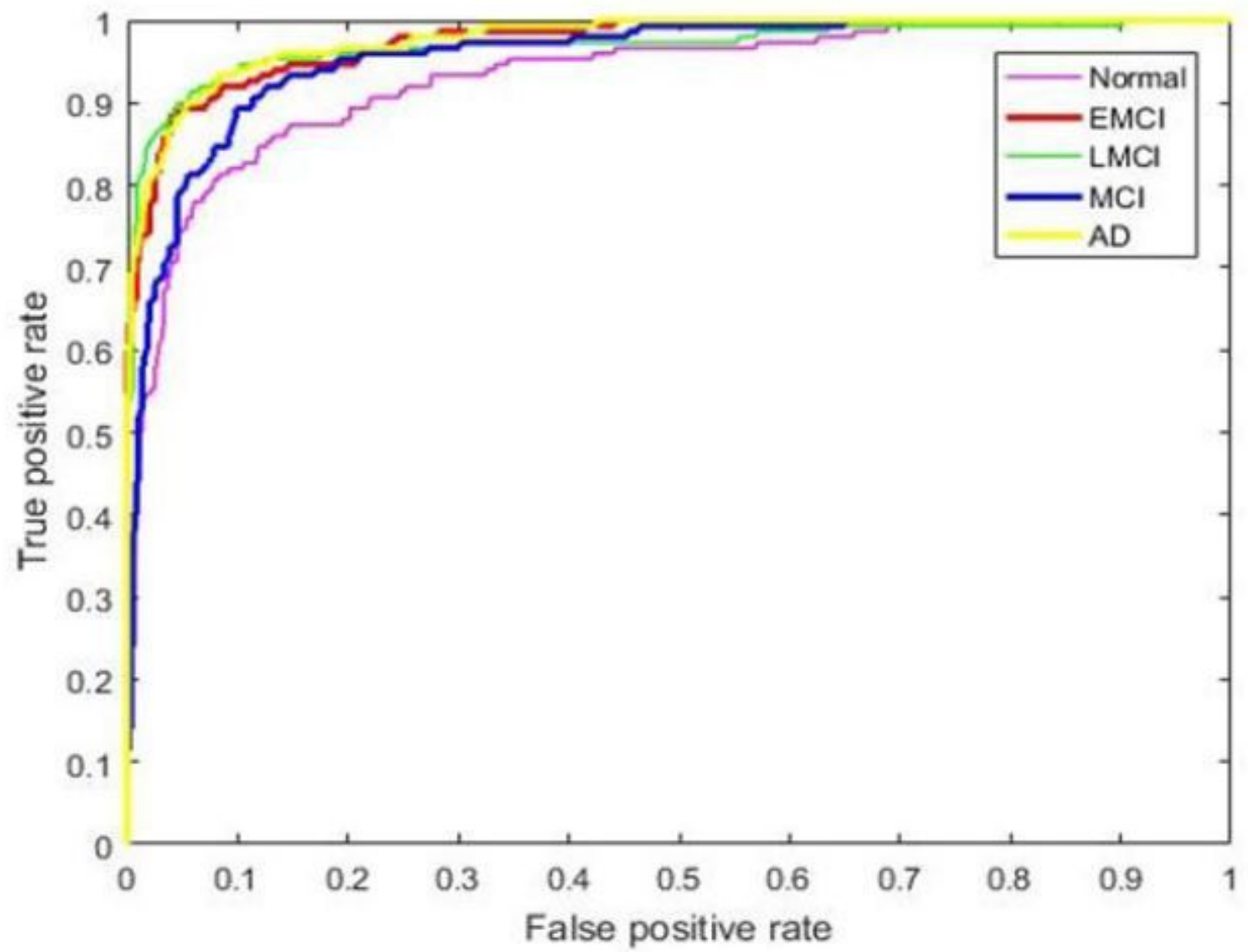

Figure 13

OC curve for "Left" ventricle using DDLT method 


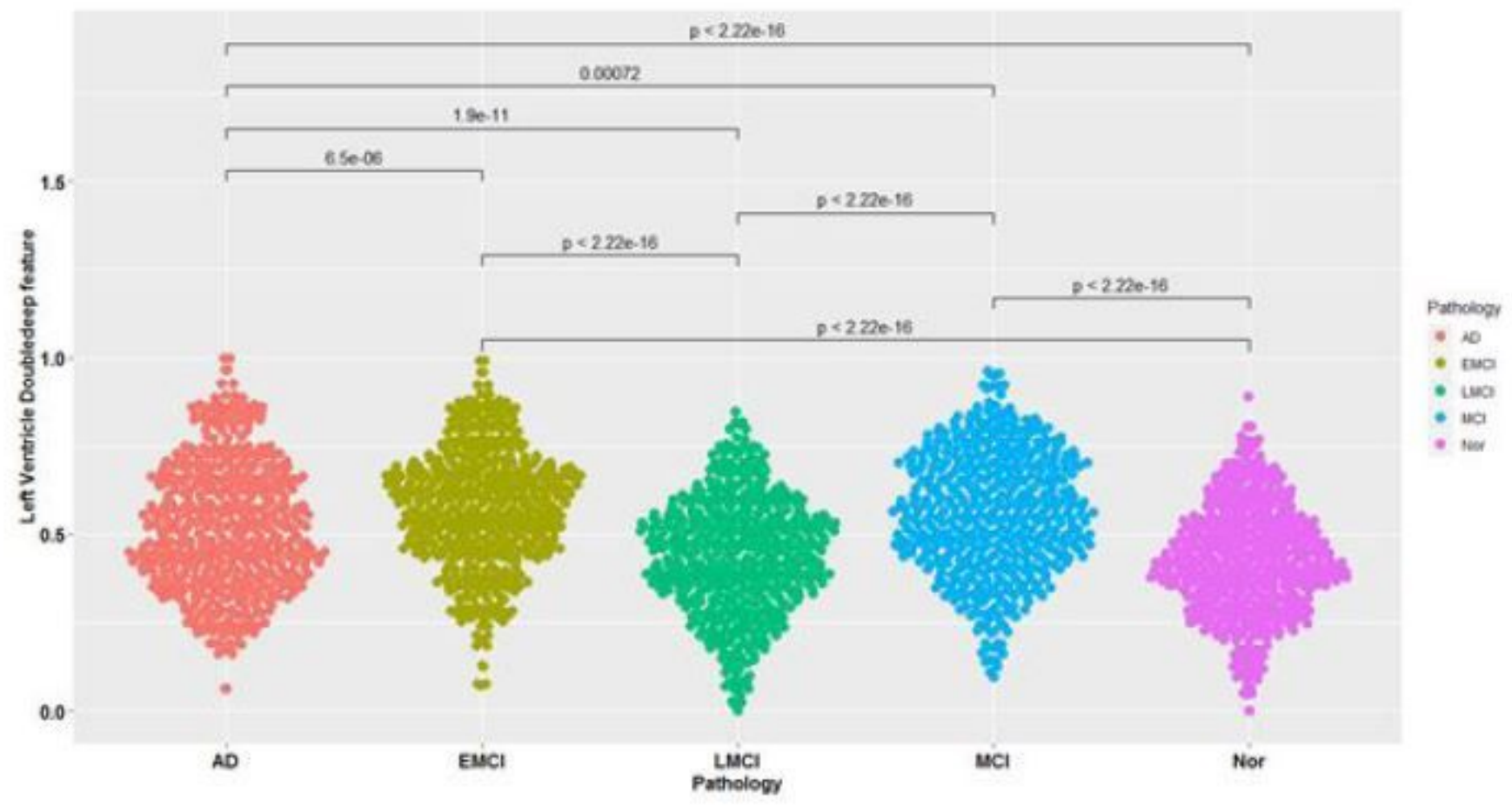

Figure 14

Statistical analysis of "Left" ventricle in normal and various severity levels 


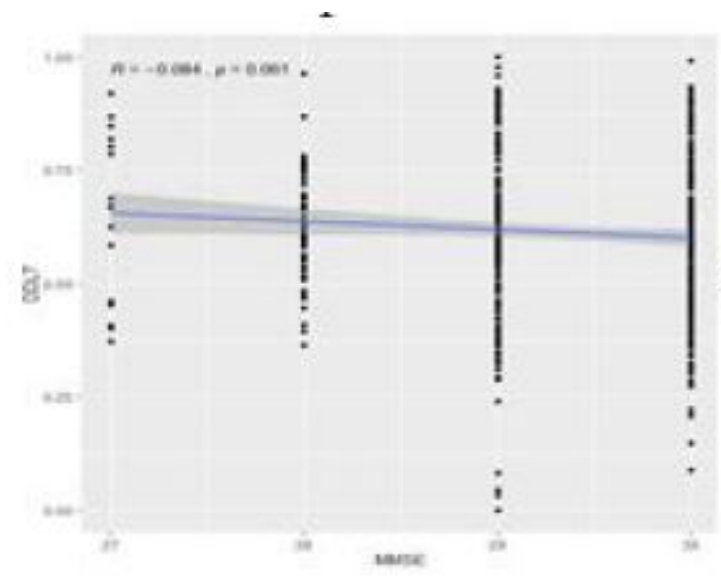

(a)

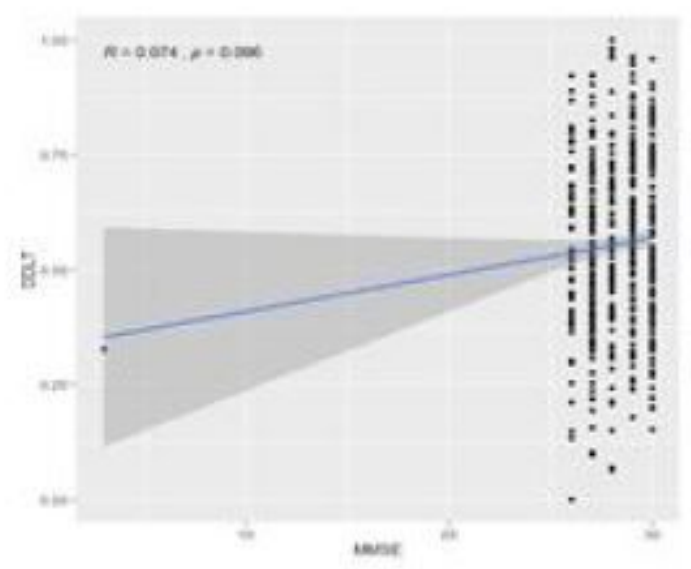

(c)

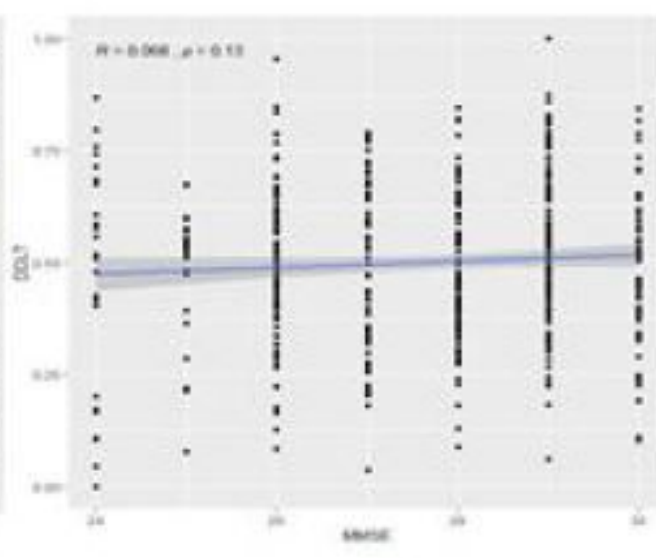

(b)

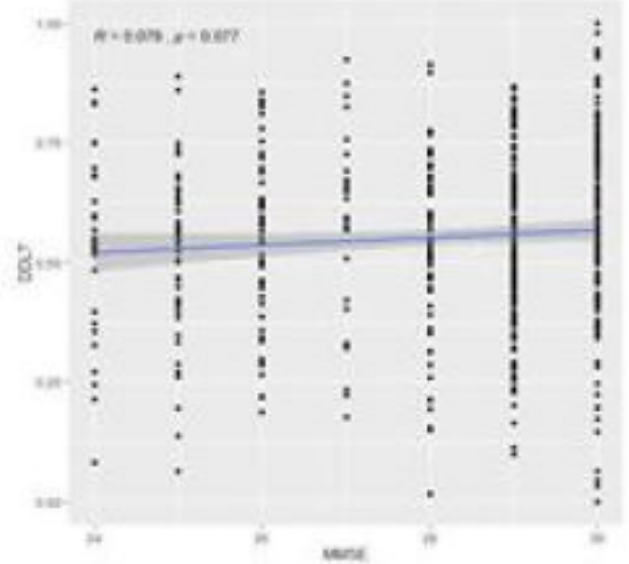

(d)

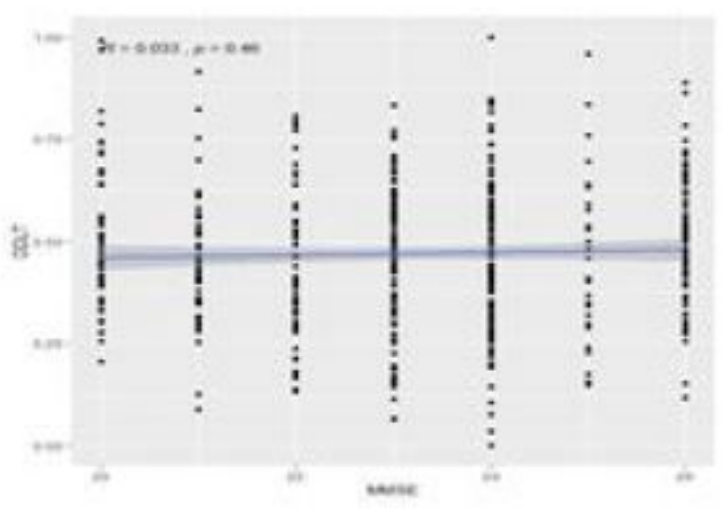

(e)

Figure 15

Clinical correlation of DDLT for (a) normal (b) EMCl (c) MCl (d) LMCland (e) AD 